\title{
Braiding Majorana Fermions and Creating Quantum Logic Gates with Vortices On a Periodic Pinning Structure
}

\author{
X. $\mathrm{Ma}^{1,2}$, C. J. O. Reichhardt ${ }^{1}$, and C. Reichhardt ${ }^{1}$ \\ 1 Theoretical Division, Los Alamos National Laboratory, \\ Los Alamos, New Mexico 87545 USA \\ 2 Department of Physics, University of Notre Dame, \\ Notre Dame, Indiana 46556 USA
}

(Dated: October 17, 2019)

\begin{abstract}
We show how vortices that support Majorana fermions when placed on a periodic pinning array can be used for vortex exchange and independent braiding by performing a series of specific moves with a probe tip. Using these braiding operations, we demonstrate realizations of a Hadamard and a CNOT gate. We specifically consider the first matching field at which there is one vortex per pinning site, and we show that there are two basic dynamic operations, move and exchange, from which basic braiding operations can be constructed in order to create specific logic gates. The periodic pinning array permits both control of the world lines of the vortices and freedom for vortex manipulation using a set of specific moves of the probe during which the probe tip strength and height remain unchanged. We measure the robustness of the different moves against thermal effects and show that the three different operations produce distinct force signatures on the moving tip.
\end{abstract}

\section{INTRODUCTION}

Manipulation of individual vortices in type-II superconductors can now be achieved with a variety of methods, including local magnetic fields ${ }^{1}$, magnetic force microscopes (MFMs) ${ }^{2} \sqrt{5}$, mechanical forces ${ }^{6}$, scanning tunneling tips ${ }^{7}$, and optically $\underline{\text { }}$. It is possible for the vortices to be moved over certain distances 1216 , entangled $\sqrt{9}$, and arranged in special positions $s^{1316} 8$. The forces induced on the tip by the motion of the vortex can also serve as a probe of the pinning properties $24 \mid 10,12$, the dynamics of individual vortices coupled to pinning $3 \sqrt{12} \mid 15$, or the creation of vortices 16 177. As advances in nanoscale fabrication continue, it will likely become possible to develop even more precise control of the vortex motion and also to manipulate multiple vortices at the same time. One promising application of vortex manipulation is to perform the braiding of Majorana fermions for quantum computing in materials for which Majorana fermions are localized in the vortex core.

Majorana fermions were first introduced by Ettore Majorana, and they have the interesting property of being their own antiparticles 18 . Currently it is unclear whether certain elementary particles in high energy physics are Majorana fermions; however, Majorana fermions in the form of quasiparticles in condensed matter systems has been a rapidly growing field, and there is now evidence that such states indeed occur in numerous systems 19]27. Another reason that such states are of interest in condensed matter is that, due to their intrinsic non-Abelian statistics, Majorana fermions can be used to support topologically protected states for quantum computation $21 / 28 \mid 29$.

Majorana fermions in condensed matter are nonAbelian anyons ${ }^{30}$ with non-trivial exchange operations which do not commute. Instead of generating a phase $2 \pi$ for bosons, $\pi$ for fermions, and arbitrary phase for
Abelian anyons, the exchange of Majorana fermions leads to a unitary transformation within the degenerate ground state manifold which does not depend on the method or details of its execution ${ }^{31132}$. The inherent nonAbelian statistics can be used to support topologicallyprotected qubits for quantum computation 33 . NonAbelian anyons were first predicted by Moore and Read to occur in the fractional quantum Hall state ${ }^{34}$, and later, Read and Green established a close connection between a two-dimensional (2D) spinless $p+i p$ superconductor and the Moore-Read quantum Hall state ${ }^{35}$, where non-Abelian statistics must be shared in the $p$-wave superconductors. Kitaev showed that non-Abelian statistics can also emerge in spinless one-dimensional (1D) superconductors ${ }^{36}$. These superconductors can contain topological phases which support exotic excitations at their boundaries and inside their topological defects 3553 . In particular, Majorana fermions can be localized at the ends of $1 \mathrm{D}$ superconductors ${ }^{36}$ and can be bonded to superconducting vortex cores in $2 \mathrm{D}$ material ${ }^{39}$. When vortices that are bonded to Majorana fermions are exchanged adiabatically, the Majorana fermions will exhibit non-Abelian statistics 40 .

In 2008, Fu and Kane proposed a physical realization of $p+i p$ superconductivity at the interface of an $s$-wave superconductor and a topological insulator ${ }^{41}$. Recent experiments using spin selective Andreev reflection verified the existence of Majorana fermions at a superconducting vortex core ${ }^{42 / 43}$. By manipulating the vortices, it is possible to manipulate the Majorana fermions (MF) trapped inside, thereby achieving transformations of the quantum states encoded by the MFs. The manipulation of MFs trapped in non-interacting (distantly separated) vortices in $p$-wave superconductors was studied recently via selfconsistent Bogolioubov-de Gennes calculations ${ }^{44}$, which showed that MF states are robust against the movement of the vortices. More recently, there have been 
proposals for the manipulation of vortex states in superconducting structures which would allow the braiding of individual vortices ${ }^{45}$ or vortex ensembles $46 / 47$ as well as other operations 485 that could be applied to quantum computing. Since there are many ways to create different types of pinning lattice structures for vortices in superconductors $53-73$ as well as numerous methods for achieving individual vortex manipulation $12|6| 8|16| 17$, a natural direction to study is what type of vortex pinning array would allow the performance of vortex exchanges that could realize the different logic gates required for topological quantum computing.

In this work we examine vortex manipulation in a topological superconductor, consisting of the interface between an $s$-wave superconductor and a topological insulator, that contains a square lattice of pinning sites in the form of blind holes. The vortex manipulation is achieved using a moving MFM probe. We propose basic operations that can independently realize vortex exchange and braiding without incorporating the world lines of other vortices. We analyze the robustness of these operations against noise and propose using the periodic potential force signals exerted on the moving probe to detect the microscopic behavior of the vortices during the different basic motions.

In addition to performing vortex exchanges, we also propose a method to braid the world lines of vortices in which the vortices end up at the same positions as in their initial state, which provides more freedom for vortex manipulation. Based on the wave function of quasiparticles in Moore-Read states, Georgiev ${ }^{74 \mid 75}$ proposed braid matrices that relate braiding operations to transformations of the quantum state, making it possible to construct braiding operations that realize quantum gates, including a Hadamard gate and a controlledNOT (CNOT) gate. Since the topological equivalence between Moore-Read states and 2D $p$-wave superconducting states has already been established ${ }^{35[40}$, we follow the braiding schemes in Refs. ${ }^{7475}$ to demonstrate our method for realizing quantum gates using vortices. We also discuss how our technique could be used in a similar scheme for skyrmion systems, based on proposals for the stabilization of bound Majorana states in skyrmions 76 -78, the pinning of skyrmions on periodic substrates ${ }^{79}$, and the manipulation of individual skyrmions 80 . There are also systems in which skyrmions and superconducting vortices are coupled $\frac{81}{\text {. }}$.

\section{SYSTEM}

In Fig. 1 we show a schematic of our system which consists of a superconductor coupled to a topological insulator. The superconductor contains a square array of blind holes that act as pinning sites capable of capturing at most one vortex. A magnetic field $\mathbf{B}$ is applied in the $z$-direction, perpendicular to the superconducting plane, with a value that corresponds to the first matching field

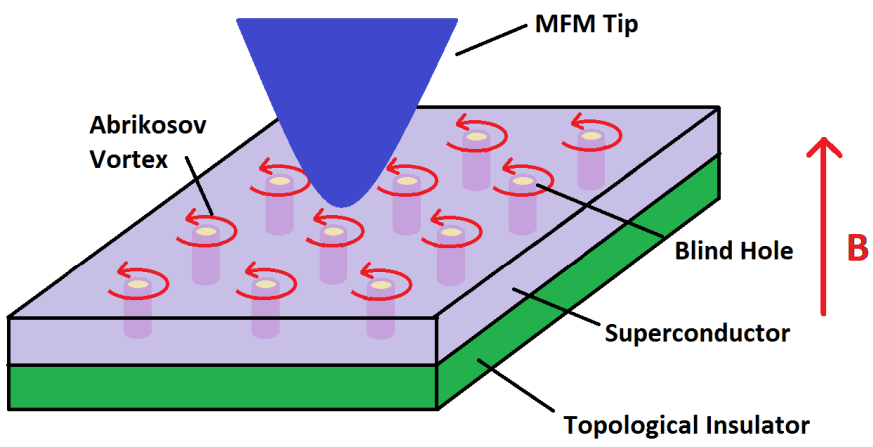

FIG. 1. Schematic of the system, consisting of a superconductor (pink) coupled to a topological insulator (green). A magnetic field $\mathbf{B}$ is applied perpendicular to the layers. The superconducting layer contains a square array of blind holes (yellow) that each capture one superconducting vortex (pink columns), and a Majorana fermion is localized inside each vortex. An MFM tip is used to manipulate individual vortices.

$B_{\phi}$ at which the number of vortices $N_{v}$ is equal to the number of pinning sites $N_{p}$. It is known from previous work that at the matching field, the vortices fill all of the pinning sites to form a commensurate structure ${ }^{54}$. A probe such as an MFM tip is used to manipulate individual vortices.

We consider a sample of size $L \times L$ with $L=40 \lambda$, where all lengths are measured in terms of the London penetration depth $\lambda$. The pinning array contains $N_{p}=400$ pinning sites arranged in a square array with a lattice constant of $a=2 \lambda$. As discussed in Refs ${ }^{47 / 82] 83}$, the hybridization strength of two vortex Majorana fermions is very small, so in our setup it can be neglected. We use the same molecular dynamics simulation technique employed in previous work on vortices in periodic pinning arrays $\frac{15}{15}$, and model the pinning sites as finite range parabolic traps with a pinning radius of $r_{p}=0.3 \lambda$ and a maximum pinning force of $F_{t r}=0.3$. The probe tip is also represented as a finite range parabolic trap with a maximum trapping force of $F_{t r}=0.65$ and a radius of $R_{t r}=0.65 \lambda$ that is translating at a velocity $V_{t r}=0.1$. The probe tip is moved slowly enough that the system remains in the adiabatic limit. At higher drives, the vortex can slip out of the probe tip, and the pinning parameters for the nonadiabatic regime have been characterized in previous work ${ }^{15}$.

The dynamics of vortex $i$ arise from the following overdamped equation of motion:

$$
\eta \frac{d \mathbf{r}_{i}}{d t}=\mathbf{F}_{i}^{v v}+\mathbf{F}_{i}^{v p}+\mathbf{F}_{i}^{t r} .
$$

Here $\eta=1$ is the damping constant and $\mathbf{r}_{i}$ is the position of vortex $i$. The vortex-vortex interaction force is $\mathbf{F}_{i}^{v v}=$ $\sum_{j=1}^{N_{v}} K_{1}\left(r_{i j} / \lambda\right) \hat{\mathbf{r}}_{i j}$, where $K_{1}$ is the modified Bessel function of the second kind, $r_{i j}=\left|\mathbf{r}_{i}-\mathbf{r}_{j}\right|, \hat{\mathbf{r}}_{i j}=\left(\mathbf{r}_{i}-\mathbf{r}_{j}\right) / r_{i j}$, and $r_{j}$ is the position of vortex $j$. We measure all forces in units of $f_{0}=\phi_{0}^{2} /\left(2 \pi \mu_{0} \lambda^{3}\right)$ where $\phi_{0}=h / 2 e$ is the flux 
quantum. The pinning force from the harmonic traps is given by $\mathbf{F}_{i}^{v p}=-\sum_{k}^{N_{p}}\left(F_{p} / r_{p}\right)\left(\mathbf{r}_{i}-\mathbf{r}_{k}^{(p)}\right) \Theta\left(r_{p}-\left|\mathbf{r}_{i}-\mathbf{r}_{k}^{(p)}\right|\right)$, where $F_{p}$ is the maximum pinning strength and $\mathbf{r}_{k}^{(p)}$ is the location of pinning site $k$. The force $\mathbf{F}^{t r}$ from the probe tip has the same form as the pinning force but the maximum probe trapping force is $F_{t r}$ and the probe trap radius $R_{t r}=0.65 \lambda$ is larger than the pinning radius. The probe tip speed is $V_{t r}=0.1$. We use a simulation time step of $\Delta t=0.02$ so that the typical time required for the probe tip to move a distance $2 \lambda$, or one pinning lattice constant, is 1000 simulation time steps. In general, after each move of the probe tip, we wait 100 simulation time steps before beginning the next move. This increases the ability of the probe tip to hold a trapped vortex.

We note that in previous work on this system $\frac{15}{15}$ we considered different types of pinning potentials such as a Gaussian trap with potential $U(r)=U_{p} \exp \left(-\kappa R_{p}^{2}\right)$. We found that the general behavior was almost the same for either harmonic or Gaussian traps with only minor changes. In general, we expect that our results will be robust as long as the pinning sites have a well defined, uniform length scale and pinning force.

\section{BASIC OPERATIONS}

\section{A. Fundamental Operations}

There are three fundamental operations that can be combined in order to create different logic gates. They are distinguished by whether the probe tip moves across a pinning site along the $\langle 10\rangle$ direction (parallel with the $x$ or $y$ axis), if the tip moves along the $\langle 11\rangle$ direction (at a $45^{\circ}$ angle from the $x$ or $y$ axis), or if the tip does not move over a pinning site at all. Due to the symmetry of the commensurate vortex lattice, when the probe tip moves over a pin along $\langle 10\rangle$, it moves the vortex in the pinning site toward its nearest neighbor a distance $a$ away. This neighbor exerts a sufficiently strong repulsive force that the vortex falls out of the probe tip and returns to its original pinned location. If instead the probe tip moves over the pin along $\langle 11\rangle$, the vortex in the pin moves toward a neighbor that is a distance $\sqrt{2} a$ away. The repulsion from this neighbor is weak enough that the probe tip is able to capture the vortex successfully and pull it out of its pinning site. The key to our proposed logic operation technique is the difference between capturing and not capturing the pinned vortices depending on whether the probe tip moves along $\langle 10\rangle$ or along $\langle 11\rangle$ as it crosses the pinning site.

The fundamental operations can be described as follows: (1) Capture. By moving the probe along $\langle 11\rangle$ as it passes over a pinning site, the probe tip captures the vortex that was in the pinning site and drags it out of the pin. (2) Move. When the probe tip contains a vortex and does not pass over a pinning site, it can move the trapped vortex over a fixed distance through the intersti-

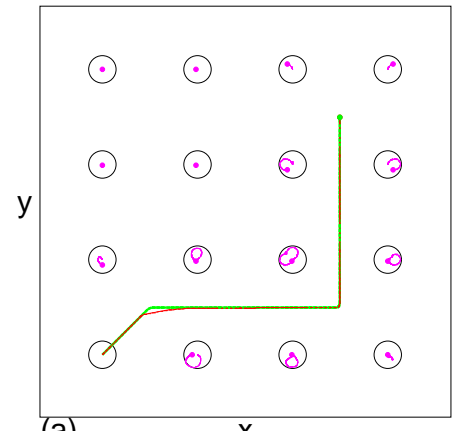

(a)

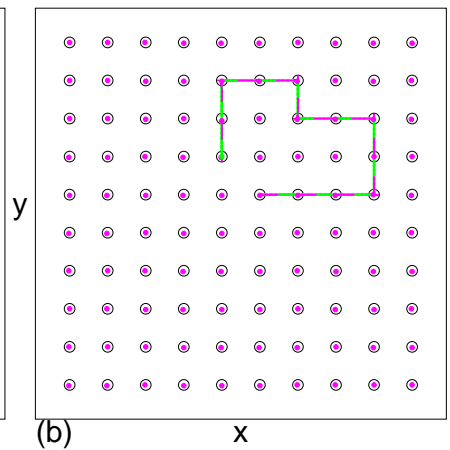

(b)
FIG. 2. Pinning site locations (open circles), vortex locations (filled pink circles), vortex trajectories (magenta lines), trapped vortex location (filled green circle), trapped vortex trajectory (red line), and probe tip trajectory (green line) for the fundamental operations. (a) The capture operation in which the probe tip moves along the $\langle 11\rangle$ direction over the pinning site and the vortex inside the pin becomes trapped by the probe tip, followed by the move operation in which the vortex is carried by the probe tip to a new location. In the illustrated trajectory, the probe tip moves the captured vortex a distance $2 a$ in the $x$ direction and $2 a$ in the $y$ direction, where $a$ is the pinning lattice constant. Only the vortex trapped by the probe tip is depinned. (b) The reposition operation where the empty probe tip moves along the $\langle 10\rangle$ direction over the pinning sites and does not capture a vortex. Individual vortices are temporarily pushed a short distance out of their pinning sites by the probe tip, but fall back into their original pinning sites as the probe tip moves away from the pin. In the illustrated trajectory, the probe is moving counterclockwise.

tial region between pinning sites. The efficiency of this operation is quantified by the distance between the position of the vortex which is supposed to move with the tip and the position of the tip at the end of these operations. (3) Reposition. By moving the probe along $\langle 10\rangle$ over a pinning site, the empty probe tip can be translated to a new position without knocking any vortices out of their pinning sites. This operation is quantified by the distance between the positions of vortices after all these operations and the positions of their original pinning sites.

In Fig. 2(a), we illustrate the capture operation followed by the move operation. Here a single vortex in the bottom left pinning site is captured by the tip which moves across the pin along the $\langle 11\rangle$ direction. The trapped vortex is then dragged a distance $2 a$ in the $x$ direction followed by a distance $2 a$ in the $y$ direction through the interstitial area between the pinning sites. The dragged vortex closely follows the tip trajectory while the other vortices remain in their pinning sites, exhibiting some rotational movement induced by the vortex-vortex interactions as the dragged vortex moves past. Figure 2(b) shows the reposition operation, where the empty trap moves over pinning sites along the $\langle 10\rangle$ direction without depinning any vortices. Individual vortices are dragged by the tip over a short distance, but 

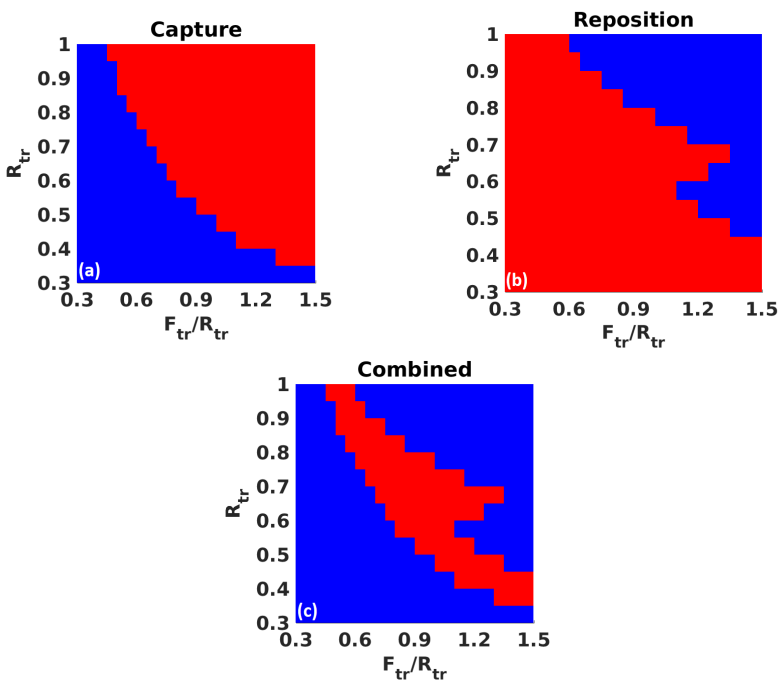

FIG. 3. Plots of the parameter regimes indicating where the fundamental operations are performed successfully (red) or unsuccessfully (blue) as a function of probe tip radius $R_{t r}$ vs probe tip spring constant $F_{t r} / R_{t r}$ in a sample with lattice constant $a=2 \lambda$ and pinning strength $F_{p}=0.3$. (a) The capture operation in which the tip crosses the pin along $\langle 11\rangle$. (b) The reposition operation in which the tip crosses the pins along $\langle 10\rangle$. (c) The combination of capture and reposition, with red indicating the region of parameter space in which both operations can be achieved simultaneously.

fall out of the tip due to the repulsion from the neighboring vortex and return to their original pinning sites, as indicated by the short linear vortex trajectories.

Experimentally, for a fixed pinning force and lattice constant $a$, the attractive strength of the probe tip can be tuned such that the capture and reposition operations can both be performed. In Fig. 3(a) we indicate the portion of the probe tip radius $R_{t r}$ and probe tip spring constant $F_{t r} / R_{t r}$ parameter space in which the capture operation is successful. The measure of these operations are converted to 0 (failure) and 1 (success) according to the quantification mentioned above with a threshold of 0.5. As the probe tip radius becomes smaller, the minimum strength of the probe required to permit capture to occur increases. For the reposition operation, Fig. 3(b) shows an opposite trend in which a decrease in the size of the probe tip radius decreases the maximum strength of the probe required to permit repositioning to occur. In Fig. 3(c), we show that there is a finite window of parameter space in which both operations can simultaneously be achieved. The widest range of tip strengths falls at a tip radius that is approximately twice the size of the pinning radius. For the remainder of this work, we consider the optimal regime with $R_{t r}=0.65$ and $F_{t r} / R_{t r}=1.0$.

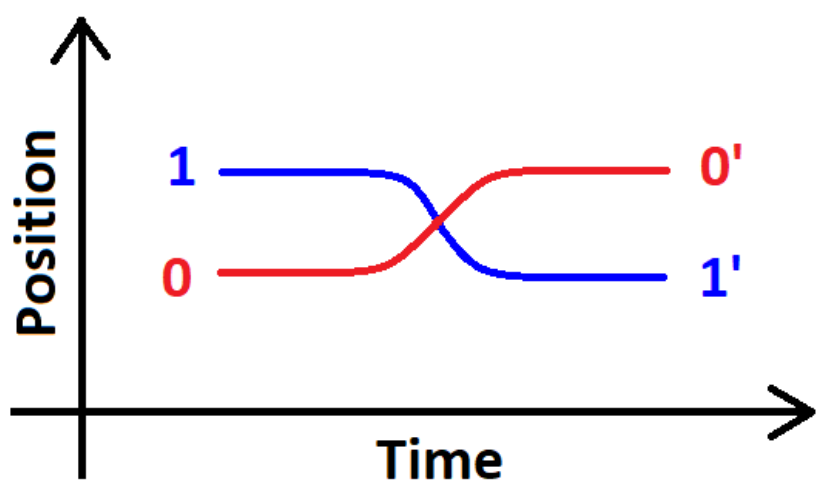

FIG. 4. The world lines as a function of position and time for an exchange operation of vortex 0 (red) with vortex 1 (blue).
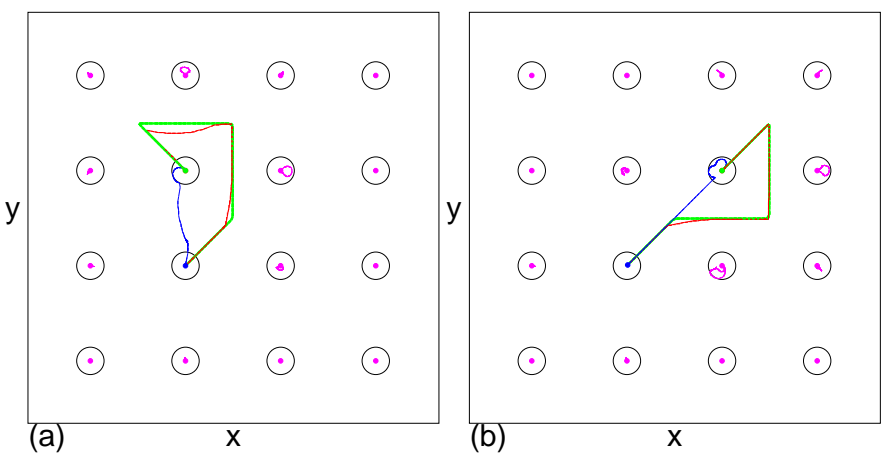

FIG. 5. Pinning site locations (open circles), vortex locations (filled circles), vortex trajectories (lines), and probe tip trajectory (green line) for two-vortex exchange operations between vortex 0 (red circle and trajectory) and vortex 1 (blue circle and trajectory). (a) A $\langle 10\rangle$ exchange. (b) A $\langle 11\rangle$ exchange.

\section{B. Exchange Operations}

Now that we have established the fundamental operations and identified optimal parameters for these operations, we consider the higher order operations needed for exchange and braiding. In Fig. 4 we show the world lines for the simple two-vortex exchange operation of vortex 0 and vortex 1 . The simplest exchange is a $\langle 10\rangle$ exchange between two vortices that are one lattice constant apart along either the $x$ or $y$ direction. As illustrated in Fig. 5(a), the probe tip first captures vortex 0 from the pin in the lower row, moves it a distance $a$ in the $+y$ direction, and then moves it a distance $a$ in the $-x$ direction. During the motion in $-x$ direction, vortex 1 was pushed down towards the empty pinning site below. Next the probe performs a reverse capture operation in which vortex 0 is pulled into the pinning site originally occupied by vortex 1 while vortex 1 is pushed by the repulsion from the vortex trapped by the probe tip into the empty pinning site that was vacated by vortex 0 . Throughout this exchange operation, the other vortices 


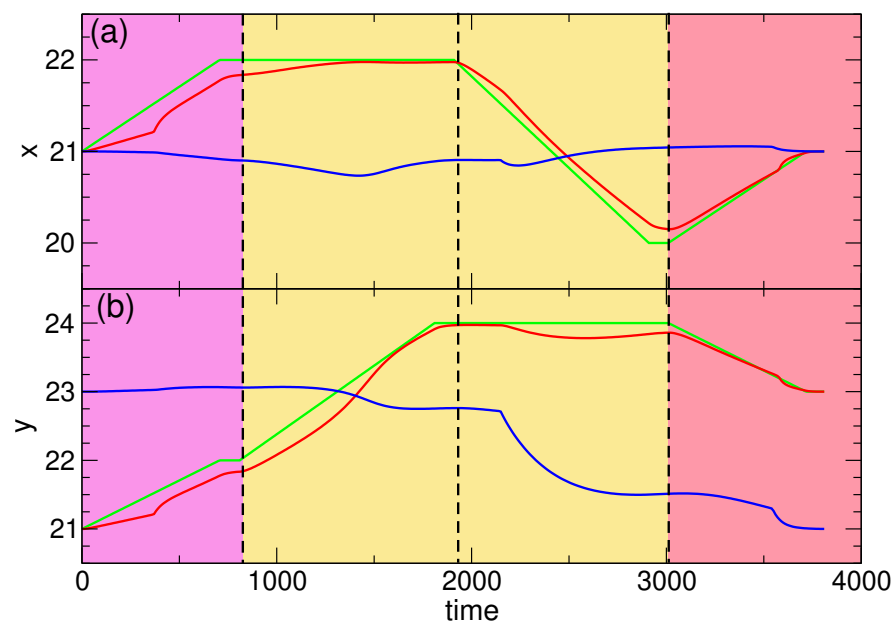

FIG. 6. The $x$ (a) and $y$ (b) positions vs time in simulation steps for vortex 0 (red), vortex 1 (blue), and the probe tip (green) for the $\langle 10\rangle$ exchange illustrated in Fig. 5(a). The vertical dashed lines delineate the four stages of probe tip motion. Magenta: capture; yellow: $\langle 10\rangle$ moves; pink: reverse capture.

remain pinned. We can characterize the path of the tip by a series of $(\theta, r / a)$ instructions where $\theta$ is the direction of motion and $r / a$ is the distance traveled in this direction in units of the lattice constant $a$. The procedure for the motion in Fig. 5(a) is $([11], \sqrt{2} / 2),([01], 1),([10], 1)$, $([1 \overline{1}], \sqrt{2} / 2)$, which, if written in terms of angles of motion from the $x$ axis, is the same as $\left(45^{\circ}, \sqrt{2} / 2\right),\left(90^{\circ}, 1\right)$, $\left(180^{\circ}, 1\right),\left(315^{\circ}, \sqrt{2} / 2\right)$. For our parameters, the move along [11] or $45^{\circ}$ takes 707 simulation time steps.

The exchange operation can also be characterized by the displacements in $x$ and $y$ of vortices 0 and 1 along with the probe tip, as shown in Fig. 6(a) and (b). Vertical dashed lines indicate the four stages of probe tip motion. Here, vortex 0 closely follows the probe tip. Since the exchange is in the $y$-direction, the $x$-positions of the vortices have the same value at the beginning and end of the operation, while the $y$-positions of vortex 0 and vortex 1 switch places by the end of the operation.

We can also perform exchange operations along the $\langle 11\rangle$ direction, as illustrated in Fig. F(b) where the probe tip again follows four stages of movement. Vortex 0 is first captured by the tip and is next moved a distance $a$ in the $+x$ direction followed by a distance $a$ in the $+y$ direction. The probe tip then performs a reverse capture in which vortex 0 is dragged into the pinning site occupied by vortex 1 , which is ejected and travels into the pinning site vacated by vortex 0 . The procedure for this exchange is $([11], \sqrt{2} / 2),([10], 1),([01], 1),([11], \sqrt{2} / 2)$. We note that in the last step we wait 400 simulation time steps to increase the stability of the operation since the $\langle 11\rangle$ exchange is more susceptible to fluctuations, as we describe in Sec. V. In Fig. $7(\mathrm{a}, \mathrm{b})$ we plot the $x$ and $y$-displacements of vortex 0 , vortex 1 and the tip as a function of time for the $\langle 11\rangle$ exchange. Vortex 0 follows

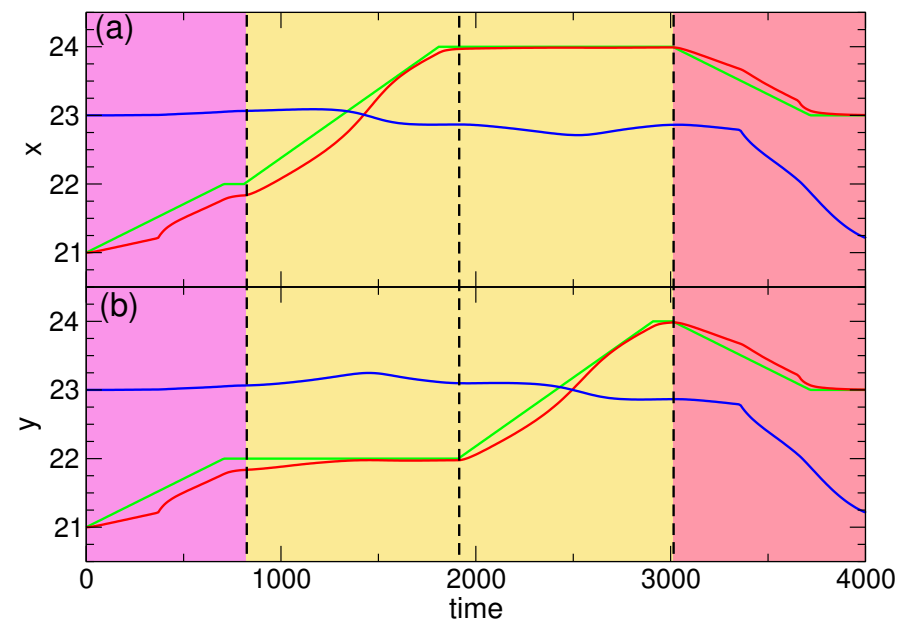

FIG. 7. The $x$ (a) and $y$ (b) positions vs time in simulation steps for vortex 0 (red), vortex 1 (blue), and the probe tip (green) for the $\langle 11\rangle$ exchange illustrated in Fig. 5(b). The vertical dashed lines delineate the four stages of probe tip motion. Magenta: capture; yellow: $\langle 10\rangle$ move; pink: reverse capture.

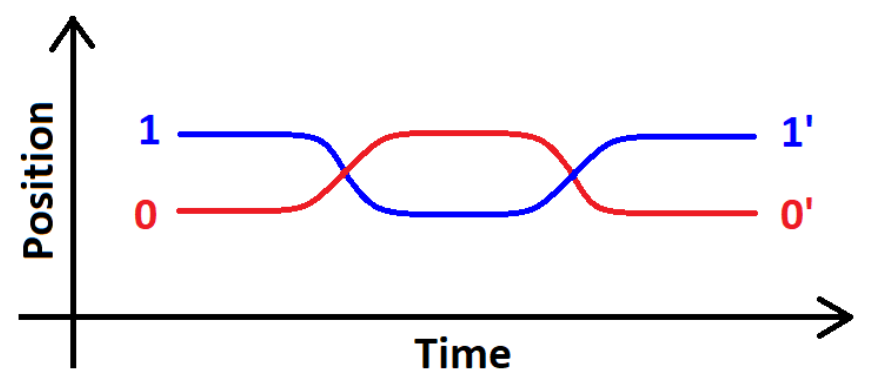

FIG. 8. The world lines as a function of position and time for a braiding operation of vortex 0 (red) and vortex 1 (blue).

the probe tip, and the final positions of vortices 0 and 1 are swapped in both the $x$ and $y$ directions by the end of the operation.

\section{Braiding Operations}

To perform a braid, the probe tip carries one vortex around one or more other vortices and then returns the original vortex to its starting position, as illustrated in the world line diagram in Fig. 8. During this operation, the vortices that are not involved in the braiding may show small perturbations in their positions but they remain localized in the pinning sites. In some cases, one of these background vortices can temporarily move out of its pinning site before returning to the same site; however, there is no exchange of these additional vortices, and their world lines do not interact with the world lines of the dragged or braided vortices. In Fig. 9(a) we illustrate the vortex positions and trajectories for a local $\langle 10\rangle$ 

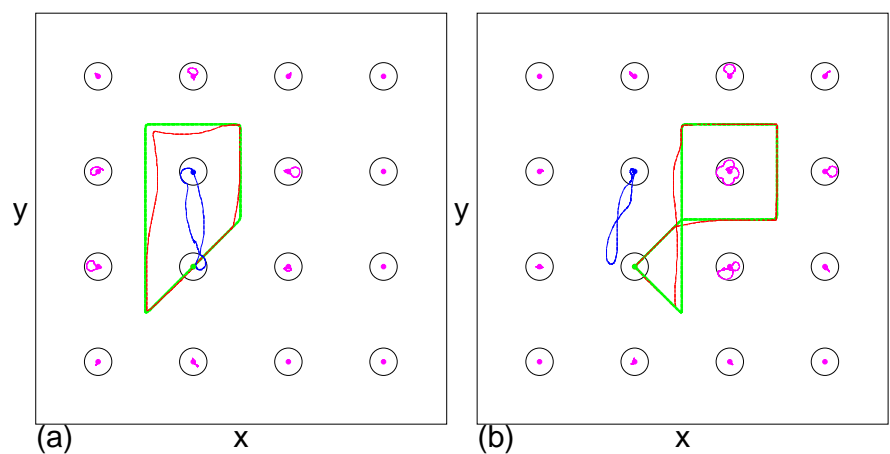

FIG. 9. Pinning site locations (open circles), vortex locations (filled circles), vortex trajectories (lines), and probe tip trajectory (green line) for braiding operations between vortex 0 (red circle and trajectory) and vortex 1 (blue circle and trajectory. (a) A $\langle 10\rangle$ braid. (b) A $\langle 11\rangle$ braid.

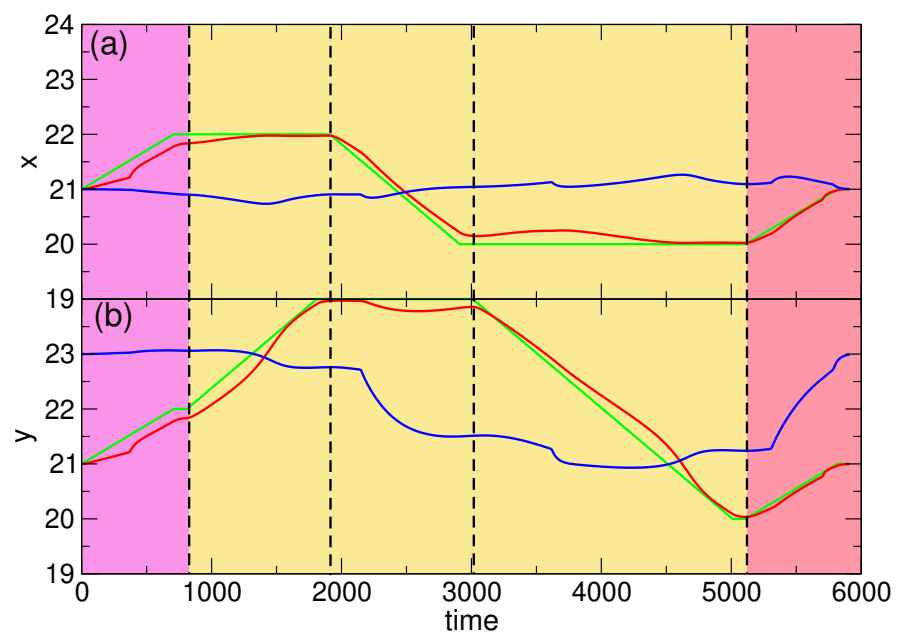

FIG. 10. The $x$ (a) and $y$ (b) positions vs time in simulation steps for vortex 0 (red), vortex 1 (blue), and the probe tip (green) for the $\langle 10\rangle$ braid operation illustrated in Fig. 9.(a). The vertical dashed lines denote the five stages of probe tip motion. Magenta: capture; yellow: $\langle 10\rangle$ moves; pink: reverse capture.

braiding operation which is achieved with 5 probe tip motion stages. Figure $10(\mathrm{a}, \mathrm{b})$ shows the $x$ and $y$ positions of vortex 0 , vortex 1 , and the probe tip during the $\langle 10\rangle$ braiding operation. In this case, the capture and reverse capture operations are performed at the same pinning site location in order to return vortex 0 to its original position. The procedure for this braiding operation is $([11], \sqrt{2} / 2),([01], 1),([\overline{1} 0], 1),([0 \overline{1}], 2),([11], \sqrt{2} / 2)$. As indicated by Fig. 9 (a), vortex 1 is actually forced out of its pinning site during the third stage, but it returns to its original pinning site during the fifth stage. All of the vortices return to their original positions at the end of the operation, in agreement with the world lines illustrated in Fig. 8. We can also perform a $\langle 11\rangle$ braiding operation using six stages of probe tip motion, as shown in Fig. 9.(b). The procedure can be written as $([11], \sqrt{2} / 2)$,

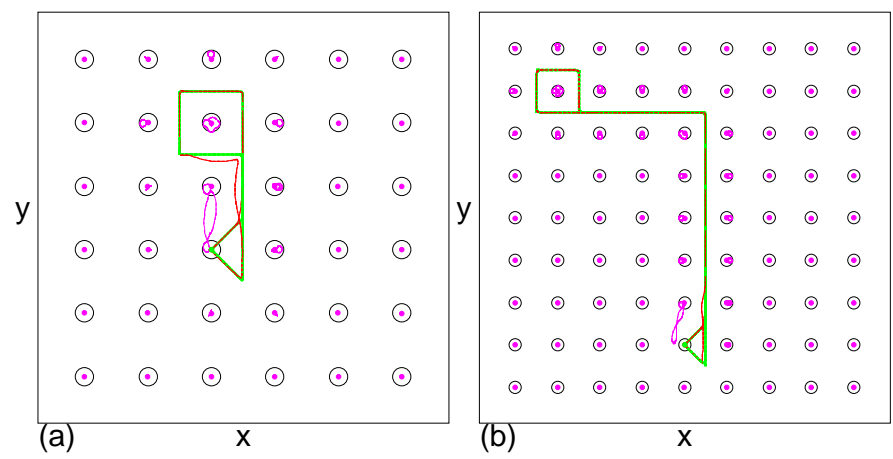

FIG. 11. Pinning site locations (open circles), vortex locations (filled circles), vortex trajectories (lines), and probe tip trajectory (green line) for braiding operations between vortex 0 (red circle and trajectory) and non-neighboring vortices. (a) Braiding of vortices that are separated by two lattice constants. (b) Braiding of distant vortices.

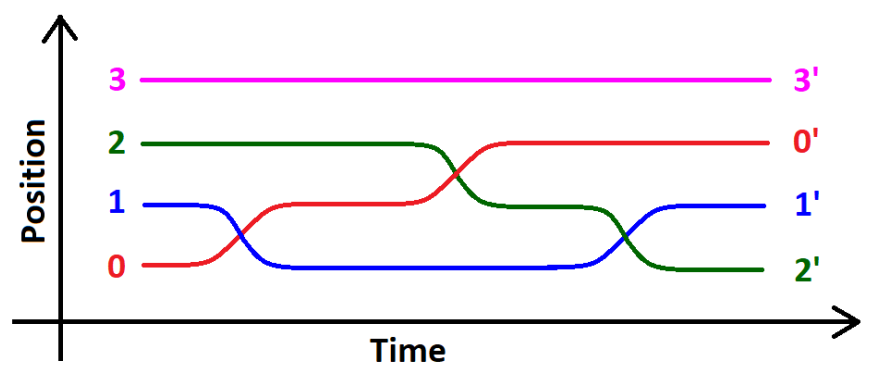

FIG. 12. The world lines as a function of position and time for a Hadamard gate involving vortex 0 (red), 1 (blue), and 2 (green), with the world line of an uninvolved vortex 3 (pink) included for comparison.

$([10], 1),([01], 1),([\overline{1} 0], 1),([0 \overline{1}], 2),([\overline{1} 1], \sqrt{2} / 2)$. In this case, a vortex that is not involved in the braiding operation makes a temporary excursion out of its pinning site before returning, but its world line does not interact with the world lines of vortices 0 or 1 .

The braiding operation can be extended to vortices that are not nearest neighbors, as shown in Fig. 11(a) where vortices that are two lattice constants away from each other are braided in a seven stage operation. The braid can be extended out to arbitrary distances as long as the coherence time of the Majorana fermions is not exceeded by the operation time. A nine step braiding operation applied to two distant vortices is illustrated in Fig. 11(b).

\section{GATE OPERATIONS}

Now that we have demonstrated the exchange and braiding operations, we show how to combine these operations in order to create Hadamard and CNOT gates. In Fig. 12 we illustrate the world lines for a Hadamard gate involving three vortices, while in Fig. 13 we plot the 


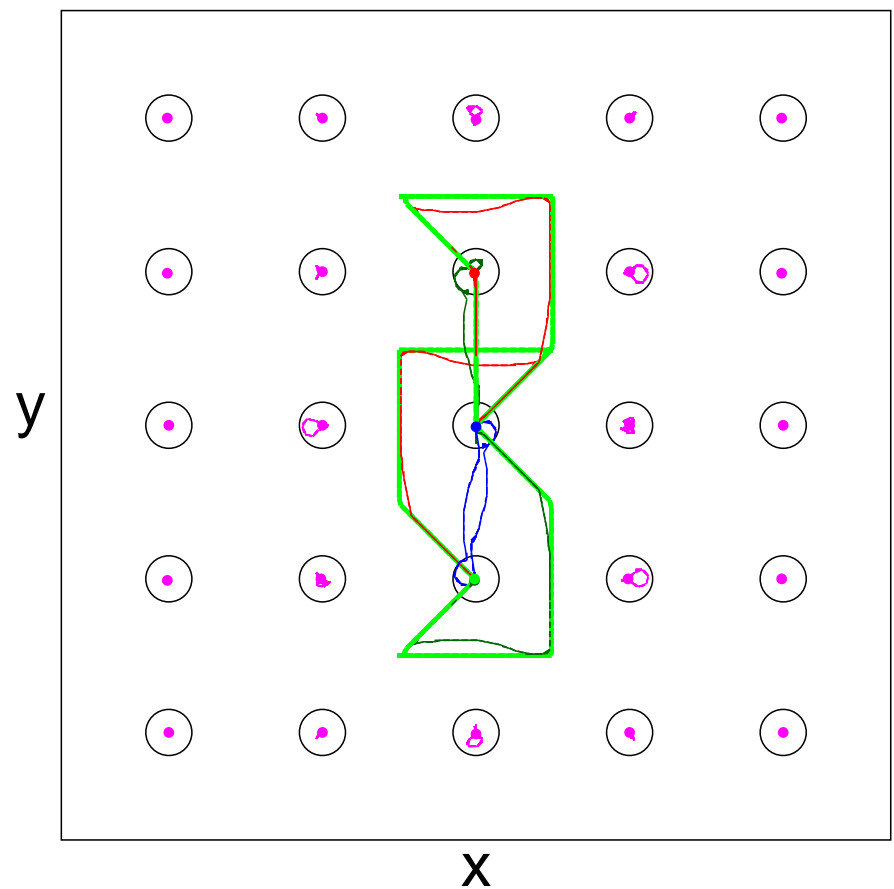

FIG. 13. Pinning site locations (open circles), vortex locations (filled circles), vortex trajectories (lines), and probe tip trajectory (green line) for a Hadamard gate involving vortex 0 (red circle and trajectory), vortex 1 (blue circle and trajectory), and vortex 2 (dark green circle and trajectory).

vortex and probe tip trajectories from a simulation of the Hadamard gate operation. The Hadamard gate creates a quantum superposition of the Majorana fermions, and our realization of this gate requires 13 stages of probe tip motion. In the first 4 stages, we perform a $\langle 10\rangle$ exchange clockwise between vortices 0 and 1 : 1 . ([11], $\sqrt{2} / 2), 2$. $([01], 1), 3 . \quad([10], 1), 4$. $([\overline{11}], \sqrt{2} / 2)$. The probe captures vortex 0 and moves it to the pinning site occupied by vortex 1 , which is ejected and travels to the pinning site originally occupied by vortex 0 . In the next 4 stages, we perform a $\langle 10\rangle$ counterclockwise exchange between vortices 0 and 2 : 5. ([11], $\sqrt{2} / 2), 6$. ([01], 1), 7. ([10], $1), 8$. $([1 \overline{1}], \sqrt{2} / 2)$. The probe captures vortex 0 and moves it to the pinning site occupied by vortex 2 , which is ejected and travels to the pinning site originally occupied by vortex 1 . In the next stage, the probe tip is repositioned to the pinning site originally occupied by vortex

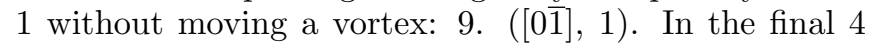
stages, we perform a $\langle 10\rangle$ exchange between vortices 2

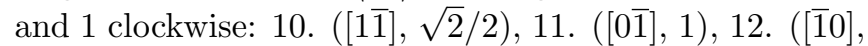
$1), 13$. ([11], $\sqrt{2} / 2)$. The probe captures vortex 2 and moves it to the pinning site originally occupied by vortex 0 . Vortex 1 is ejected from this pinning site and returns to the pinning site it originally occupied. In Fig. 14 we plot the $x$ and $y$ positions versus time in simulation steps for vortices 0,1 , and 2 along with the probe tip for the successful simulated operation of the Hadamard gate.

Now that we have shown the achievement of a single

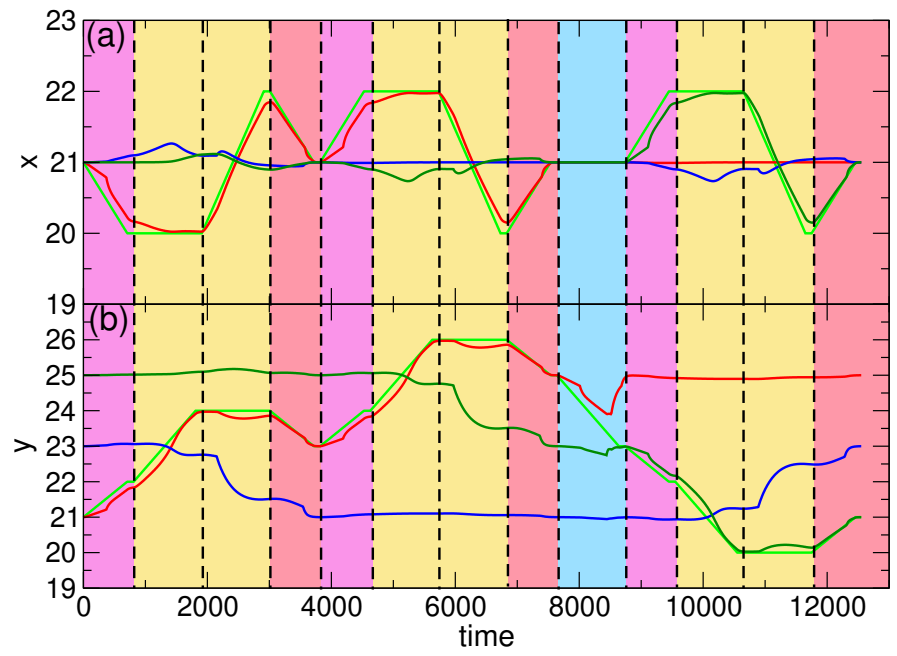

FIG. 14. The $x$ (a) and $y$ (b) positions vs time in simulation steps for vortex 0 (red), vortex 1 (blue), vortex 2 (dark green), and the probe tip (light green) for the Hadamard gate operation illustrated in Fig. 13 . The vertical dashed lines indicate the 13 stages of probe tip motion. Magenta: capture; yellow: $\langle 10\rangle$ moves; blue: repositioning of probe; pink: reverse capture.

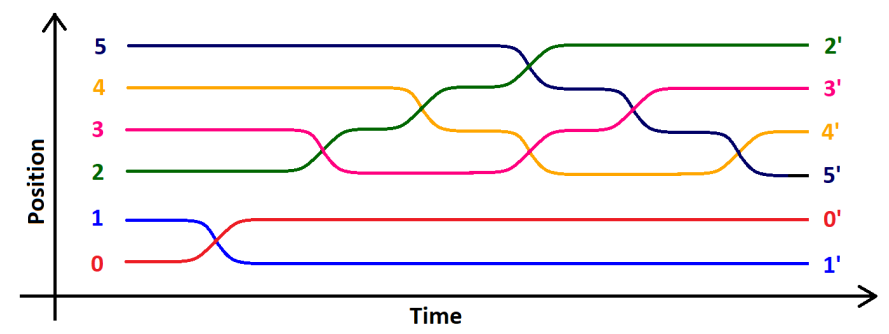

FIG. 15. The world lines as a function of position and time for a CNOT gate involving vortex 0 (red), 1 (blue), 2 (dark green), 3 (pink), 4 (orange), and 5 (violet).

qubit gate by a vortex braiding operation, we demonstrate a CNOT gate as an example of a 2-qubit gate. In Fig. 15(a) we show the world lines for a CNOT gate involving six vortices. We select 6 vortices that are in a vertical line, as shown in Fig. 16 where we illustrate the motion of the vortices and the probe tip. The CNOT operation requires 31 stages of probe motion. In the first 4 stages, we perform a $\langle 10\rangle$ exchange between vortices 0 and 1: 1. ([11], $\sqrt{2} / 2), 2$. $([01], 1), 3 .([\overline{1} 0], 1), 4 .([1 \overline{1}]$, $\sqrt{2} / 2)$. The probe captures vortex 0 and moves it to the pinning site occupied by vortex 1 , which is ejected and travels to the pinning site originally occupied by vortex 0 . In the next stage, the probe tip is repositioned to the pinning site occupied by vortex 2 without moving a vortex: 5 . $([01], 1)$. During the next 4 stages, we perform a $\langle 10\rangle$ exchange between vortices 2 and $3: 6$. ([11], $\sqrt{2} / 2)$, 7. $([01], 1), 8$. $([10], 1), 9$. ([11],$\sqrt{2} / 2)$. The probe captures vortex 2 and moves it to the pinning site occupied by vortex 3 , which is ejected and travels to the pinning 


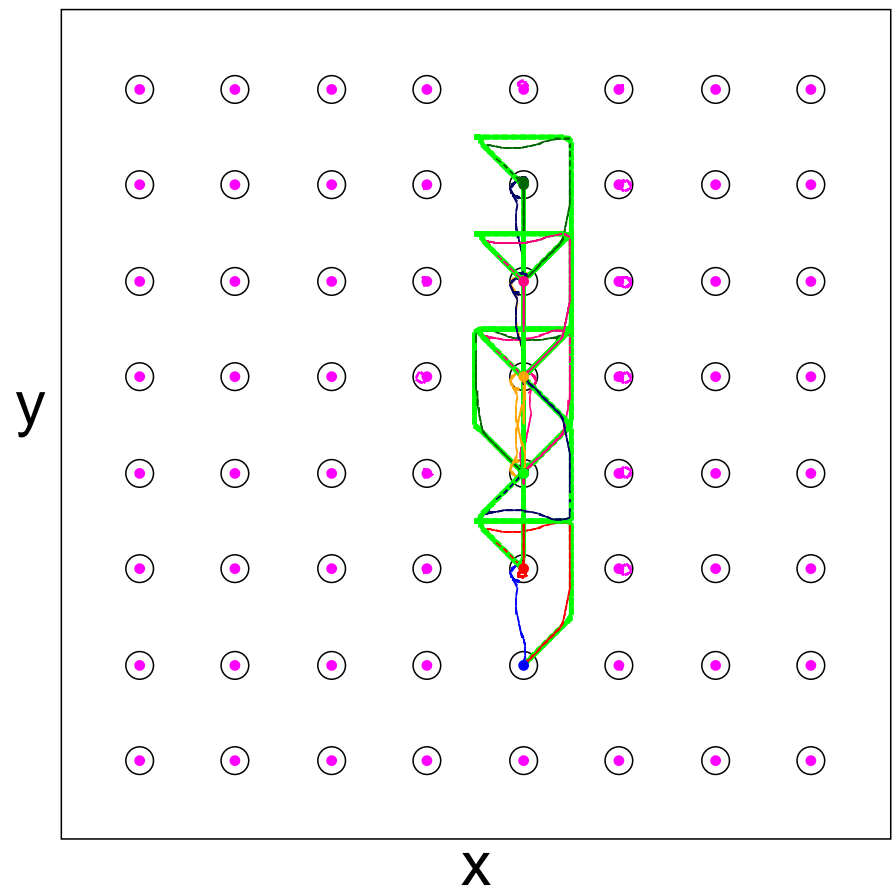

FIG. 16. Pinning site locations (open circles), vortex locations (filled circles), vortex trajectories (lines), and probe tip trajectory (green line) for a CNOT gate involving vortex 0 (red circle and trajectory), 1 (blue circle and trajectory), 2 (green circle and trajectory), 3 (pink circle and trajectory), 4 (orange circle and trajectory), and 5 (violet circle and trajectory).

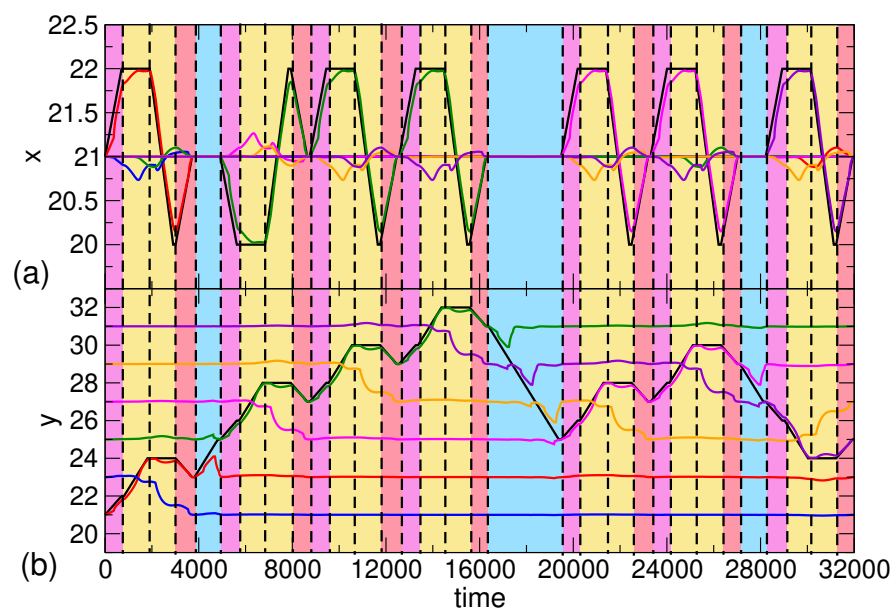

FIG. 17. The $x$ (a) and $y$ (b) positions vs time in simulation steps for vortex 0 (red), vortex 1 (blue), vortex 2 (dark green), vortex 3 (pink), vortex 4 (orange), vortex 5 (violet), and the probe tip (black) for the CNOT gate operation illustrated in Fig. 16 The vertical dashed lines indicate the 31 stages of probe tip motion. Magenta: capture; yellow: $\langle 10\rangle$ moves; blue: repositioning of probe; pink: reverse capture. site originally occupied by vortex 2 . In the next 4 stages we perform a $\langle 10\rangle$ exchange between vortices 2 and 4 : 10. $([11], \sqrt{2} / 2), 11 .([01], 1), 12 .([\overline{1} 0], 1), 13 .([1 \overline{1}]$, $\sqrt{2} / 2)$. The probe captures vortex 2 and moves it to the pinning site occupied by vortex 4 , which is ejected and travels to the pinning site originally occupied by vortex 3 . The next 4 stages are a $\langle 10\rangle$ exchange between vortices 2 and 5: 14. ([11], $\sqrt{2} / 2), 15 .([01], 1), 16$. ([10], $1), 17$. ([1] $], \sqrt{2} / 2)$. The probe captures vortex 2 and moves it to the pinning site occupied by vortex 5 , which is ejected and travels to the pinning site originally occupied by vortex 4 . In the next stage, the probe tip is repositioned to the pinning site originally occupied by vortex 2 without moving a vortex: 18 . ([0]1], 3). The next 4 stages are a $\langle 10\rangle$ exchange between vortices 3 and

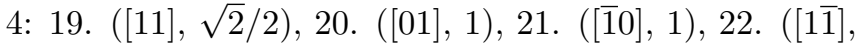
$\sqrt{2} / 2)$. The probe captures vortex 3 and moves it to the pinning site originally occupied by vortex 3 . This returns vortex 3 to its starting location and causes the ejection of vortex 4 , which travels to the pinning site originally occupied by vortex 2 . The following 4 stages are a $\langle 10\rangle$ exchange between vortices 3 and 5: 23. ([11], $\sqrt{2} / 2), 24$. $([01], 1), 25$. ([1]0], 1), 26. ([1] $], \sqrt{2} / 2)$. The probe captures vortex 3 and moves it to the pinning site originally occupied by vortex 4 . This causes the ejection of vortex 5 , which travels to the pinning site originally occupied by vortex 3 . In the next stage, the probe tip is repositioned to the pinning site originally occupied by vortex 3 without moving a vortex: 27 . $([0 \overline{1}], 1)$. During the final four stages, a $\langle 10\rangle$ exchange is performed between vortices 4

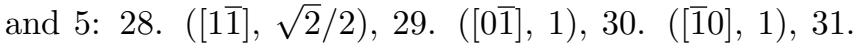
$([11], \sqrt{2} / 2)$. The probe captures vortex 5 and moves it to the pinning site originally occupied by vortex 2 . This causes the ejection of vortex 4 , which travels to the pinning site originally occupied by vortex 3 . In Fig. 17 we plot the $x$ and $y$ positions of vortices 0 through 5 and the probe tip during the simulated operation of the CNOT gate during a time span of 32000 simulation time steps.

We note that other quantum gates can be realized following similar vortex braiding methods, and thus complicated logic operations can be achieved. In principle it would be possible to perform the CNOT and Hadamard operations using only a $1 \mathrm{D}$ chain of pinned vortices. In that case, however, it is possible that other unconfined vortices outside of the chain could occupy random positions that might interfere with the logic operations. By trapping all of the vortices in a periodic pinning array, we can guarantee that a minimum distance is maintained between the passive background vortices and the active vortices that are involved in the logic operation.

\section{ROBUSTNESS}

We next analyze the robustness of our logic operations against noise. To achieve this, we add thermal fluctuations to the motion of the vortices and compare this 

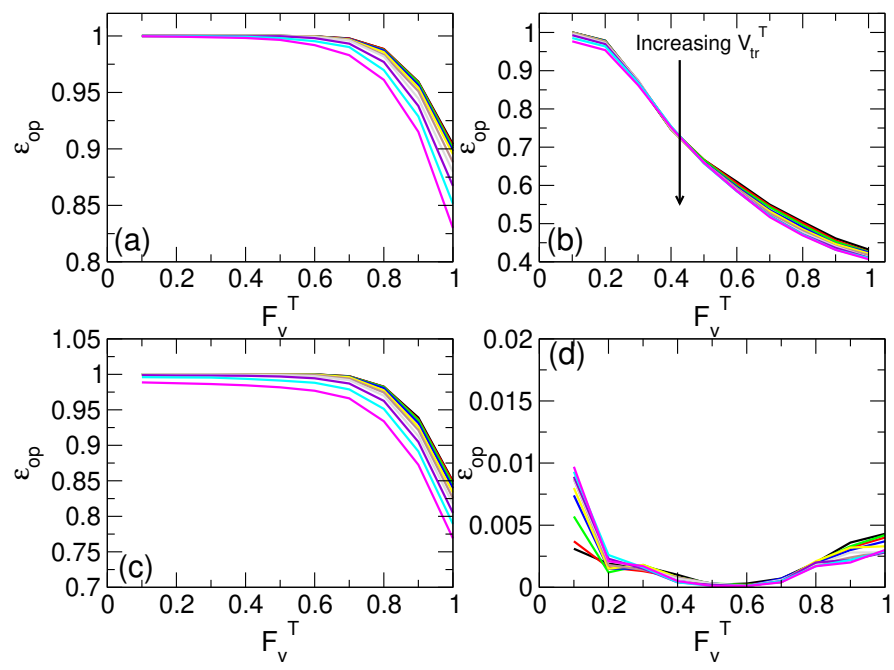

FIG. 18. The fidelity $\epsilon_{\mathrm{op}}$ of the operations vs the magnitude $F_{v}^{T}$ of the thermal noise on the vortices for varied probe tip fluctuation magnitudes $V_{t r}^{T}=0.02$ (black), 0.04, 0.06, 0.08, $0.10,0.12,0.14,0.16,0.18$, and 0.20 (magenta). (a) The capture operation. (b) The reposition operation. (c) The $\langle 10\rangle$ exchange. (d) The $\langle 11\rangle$ exchange, which shows a strong sensitivity to noise.

with the addition of thermal motions to the probe tip itself. In all cases, the probe tip moves at an average velocity of $V_{t r}=0.1$ and we fix $F_{t r}=0.3$. The thermal fluctuations $F_{i}^{T}$ have the properties $\left\langle F_{i}^{T}\right\rangle=0$ and $\left\langle F_{i}^{T}(t) F_{j}^{T}\left(t^{\prime}\right)\right\rangle=2 \eta k_{B} T \delta_{i j} \delta\left(t-t^{\prime}\right)$. We consider fluctuations ranging from $V_{t r}^{T}=0.02$ to $V_{t r}^{T}=0.2$ in intervals of $\Delta V_{t r}^{T}=0.02$ for the probe tip, and fluctuations ranging from $F_{v}^{T}=0.1$ to $F_{v}^{T}=1.0$ in intervals of $\Delta F_{v}^{T}=0.1$ for the vortices. We perform each logic operation $O_{L}=10000$ times in the presence of the fluctuations and compute the fidelity $\epsilon_{\mathrm{op}}=O_{s} / O_{L}$, given by the ratio of the number of successful operations $O_{s}$ to the total number of attempted operations $O_{L}$. In Fig. 18(a) we plot $\epsilon_{\mathrm{op}}$ versus vortex thermal noise magnitude $F_{v}^{T}$ for the capture operation performed at different values of the tip noise $V_{t r}^{T}$. The fidelity remains close to $\epsilon_{\mathrm{op}}=1$ for $F_{v}^{T}<0.8$ and begins to drop as the vortex thermal noise increases above this value, while the tip noise has only a very weak effect on the fidelity. In Fig. 18(b) we show $\epsilon_{\text {op }}$ versus $F_{v}^{T}$ for the reposition operation, which depends more sensitively on the vortex thermal noise. For $F_{v}^{T}<0.2, \epsilon_{\mathrm{op}} \approx 1$; however, by the time $F_{v}^{T}=0.6$, the fidelity has dropped to a value of less than $50 \%$. The reposition operation is also only weakly affected by tip fluctuations. Figure 18(c) shows the fidelity versus $F_{v}^{T}$ for the $\langle 10\rangle$ exchange operation, which has $\epsilon_{\mathrm{op}} \approx 1$ when $F_{v}^{T}<0.8$. In Fig. 18(d), the plot of $\epsilon_{\mathrm{op}}$ versus $F_{v}^{T}$ for the $\langle 11\rangle$ exchange operation, which functions well at zero temperature, shows that the fidelity drops nearly to zero as soon as vortex thermal fluctuations are added. This extreme sensitivity to noise makes the $\langle 11\rangle$ exchange operation poorly suited for use in logic gates, and this is

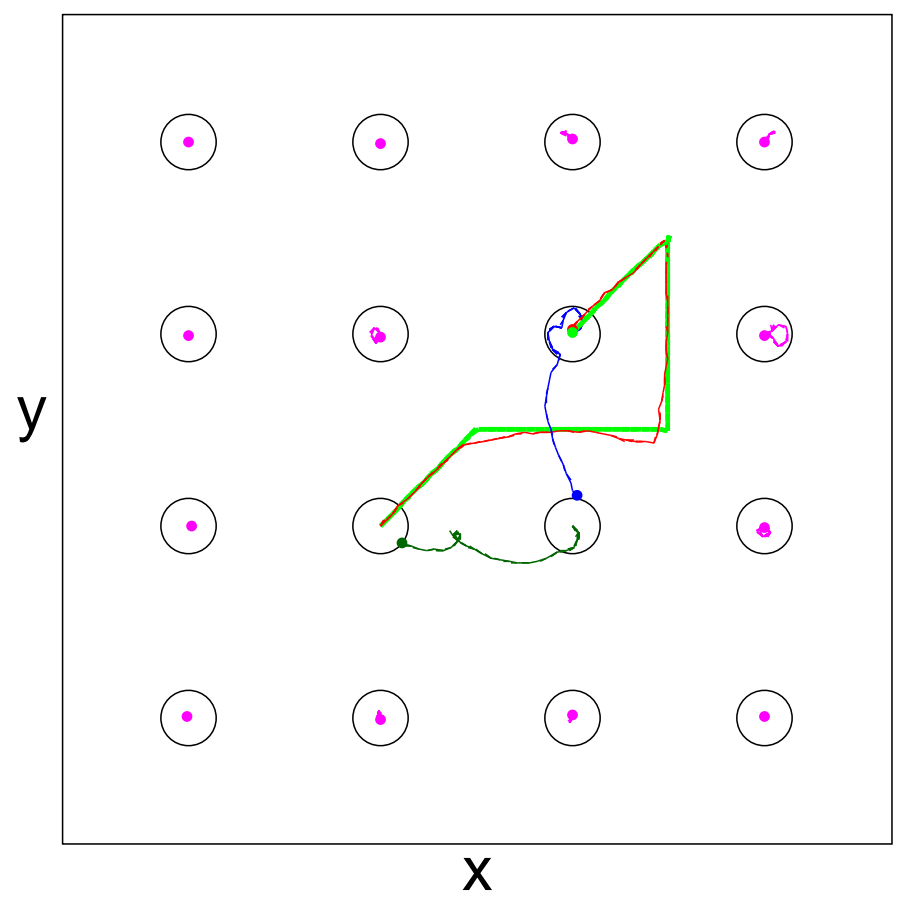

FIG. 19. Pinning site locations (open circles), vortex locations (filled circles), vortex trajectories (lines), and probe tip trajectory (green line) for the $\langle 11\rangle$ exchange operation performed at a finite temperature of $F_{v}^{T}=0.1$. Vortex 0 (red circle and trajectory) and vortex 1 (blue circle and trajectory) should be the only two vortices participating in the exchange; however, due to the thermal fluctuations, vortex 2 (dark green circle and trajectory) is able to depin and interfere with the operation.

why we did not employ this operation in our proposed gates.

The origin of the extreme noise sensitivity of the $\langle 11\rangle$ exchange operation is illustrated in Fig. 19, where we highlight the trajectories at $F_{v}^{T}=0.1$ of the probe tip, vortices 0 and 1 , and a third vortex that is supposed to remain in the passive background of the operation. When $F_{v}^{T}=0$, as in Fig. 5(b), the third vortex does not interfere with the operation; however, once thermal noise is added, the motion of vortex 1 can cause the third vortex to depin and move into the pinning site originally occupied by vortex 0 , resulting in a failure of the exchange. We find that a similar effect occurs for the $\langle 11\rangle$ braiding operation as well. This suggests that only the $\langle 10\rangle$ exchange and braiding operations, which are not sensitive to the weak shear mode of the vortex lattice, should be used for gate creation if thermal noise will be significant.

\section{FORCE SIGNALS}

In our simulations, we can trace the complete vortex trajectory during the operations; however, in experiments performed using an MFM or other tip, it would be valuable to be able to determine whether the operation 


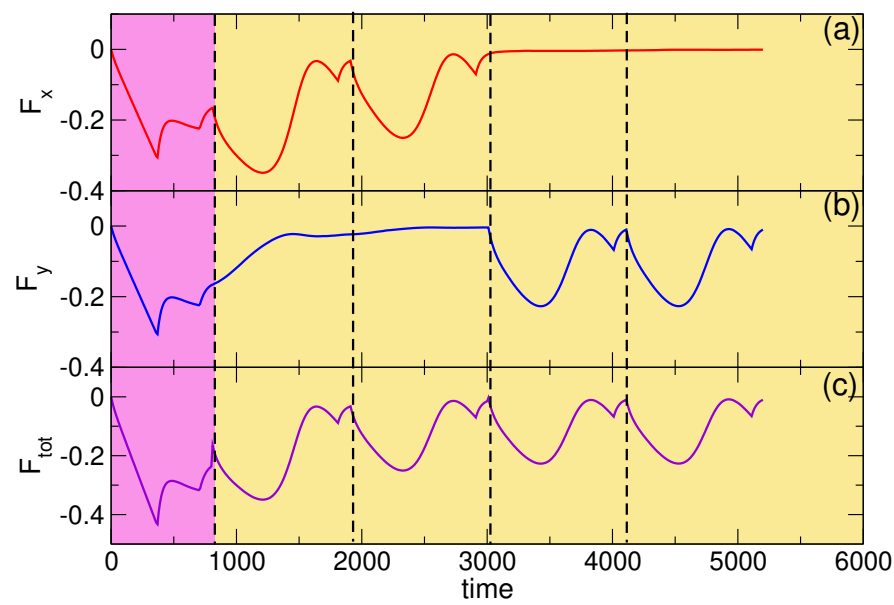

FIG. 20. Probe tip force signatures $F_{x}$ (a), $F_{y}$ (b), and $F_{\text {tot }}$ (c) vs time in simulation steps for the capture (magenta) and $\langle 10\rangle$ move (yellow) operations illustrated in Fig. 2(a).

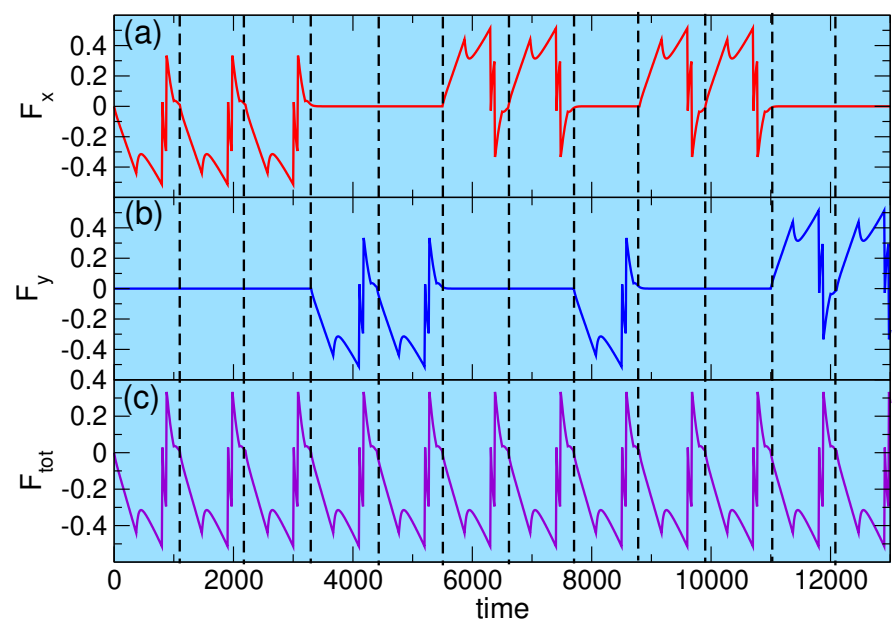

FIG. 21. Probe tip force signatures $F_{x}$ (a), $F_{y}$ (b), and $F_{\text {tot }}(\mathrm{c})$ vs time in simulation steps for the reposition (blue) operations illustrated in Fig. 2(b).

was successful by simply measuring the force fluctuations experienced by the tip. We find that the operations produce a specific set of force patterns on the probe tip that can be divided into five groups. In the first group, the probe tip pulls a vortex out of a pinning site. In the second group, the probe tip transports a vortex symmetrically between two pinned vortices, which remain pinned. The third group is for asymmetric transport of a vortex by the probe tip between two pinned vortices, where one of the pinned vortices has only a single pinned neighboring vortex perpendicular to the direction in which the probe tip is moving. This vortex is depinned by the passage of the vortex trapped by the probe tip. In the fourth group, the probe tip deposits its trapped vortex in a pinning site. The fifth group is when the probe tip is repositioned and does not carry a trapped vortex. The characteristic force signals of these five groups of patterns can

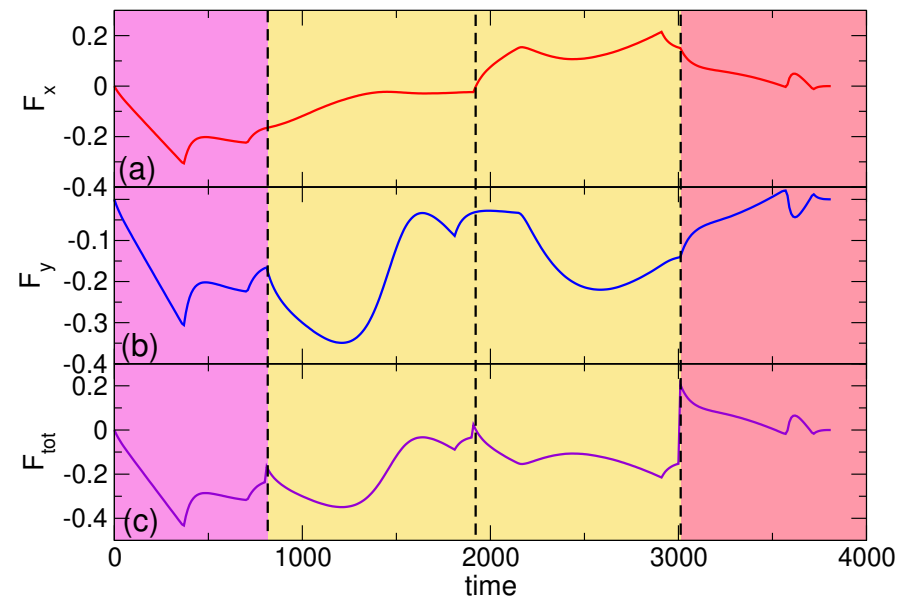

FIG. 22. Probe tip force signatures $F_{x}$ (a), $F_{y}$ (b), and $F_{\text {tot }}$ (c) vs time in simulation steps for the $\langle 10\rangle$ exchange operation illustrated in Figs. 5(a) and 6. Magenta: capture; yellow: $\langle 10\rangle$ moves; pink: reverse capture.

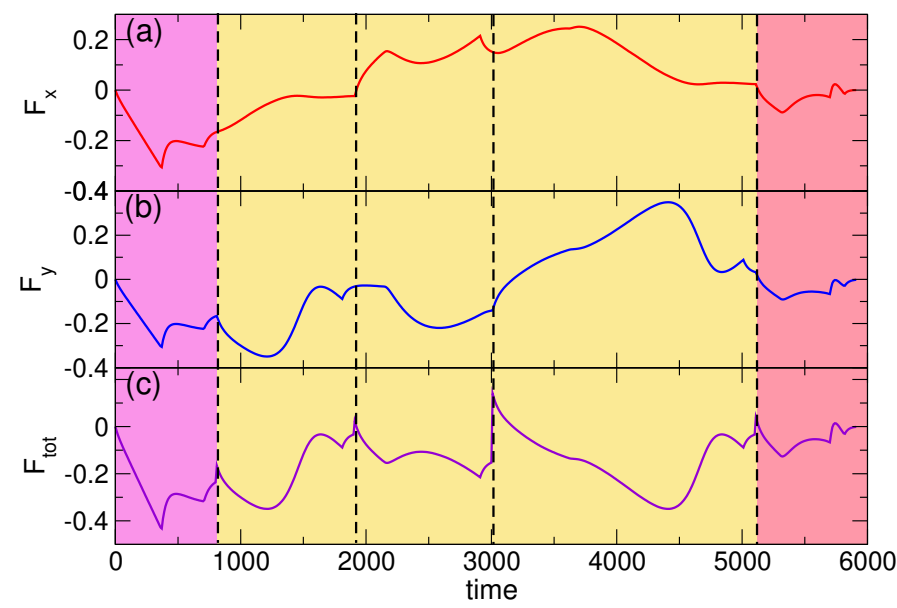

FIG. 23. Probe tip force signatures $F_{x}$ (a), $F_{y}$ (b), and $F_{\text {tot }}$ (c) vs time in simulation steps for the $\langle 10\rangle$ braiding operation illustrated in Figs. 9(a) and 10 Magenta: capture; yellow: $\langle 10\rangle$ moves; pink: reverse capture.

be used to determine whether the attempted operation was successfully completed. Additionally, a measurement of the $x$ and $y$ components of the forces make it possible to reconstruct the trajectory of the probe tip in the experiment. In Fig. 20(a,b,c) we plot $F_{x}, F_{y}$, and the total force $F_{\text {tot }}$ on the probe tip versus time for the capture and move operations illustrated in Fig.2(a), while in Fig. 21 (a,b,c) we show the same for the reposition operations from Fig. 2(b). The capture signal, highlighted in pink in Fig. 20, is an abrupt downward spike in all three quantities, while the move signal, highlighted in yellow in Fig. 20, has a sinusoidal form in the direction of motion. The repositioning signal, shown in blue in Fig. 21, has a double spike feature.

In Fig. 22 we plot $F_{x}, F_{y}$, and $F_{\text {tot }}$ versus time for the $\langle 10\rangle$ exchange operation, and in Fig. 23 we plot the same 


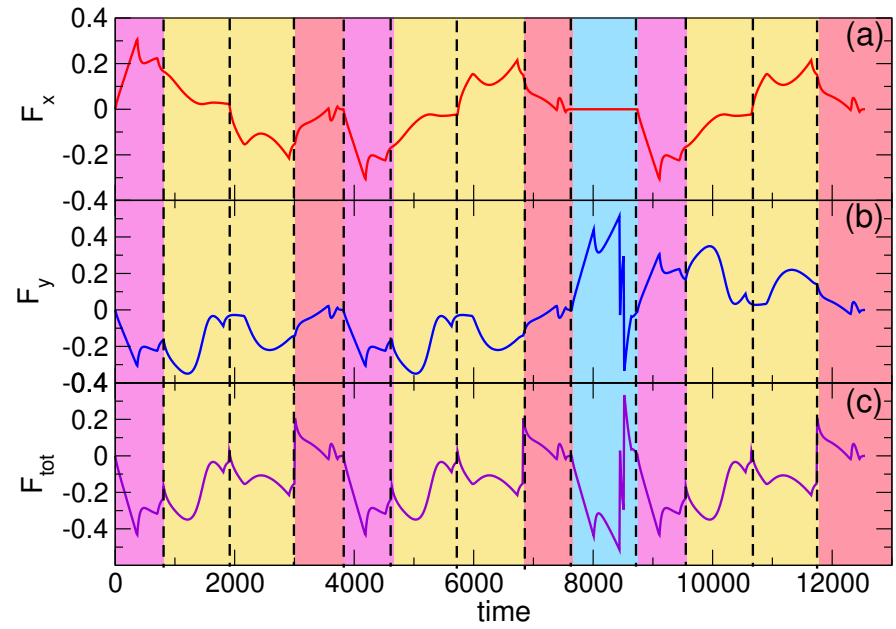

FIG. 24. Probe tip force signatures $F_{x}(\mathrm{a}), F_{y}(\mathrm{~b})$, and $F_{\text {tot }}(\mathrm{c})$ vs time in simulation steps for the Hadamard gate operation illustrated in Figs. 13 and 14 . Magenta: capture; yellow: $\langle 10\rangle$ moves; pink: reverse capture; blue: repositioning of probe.

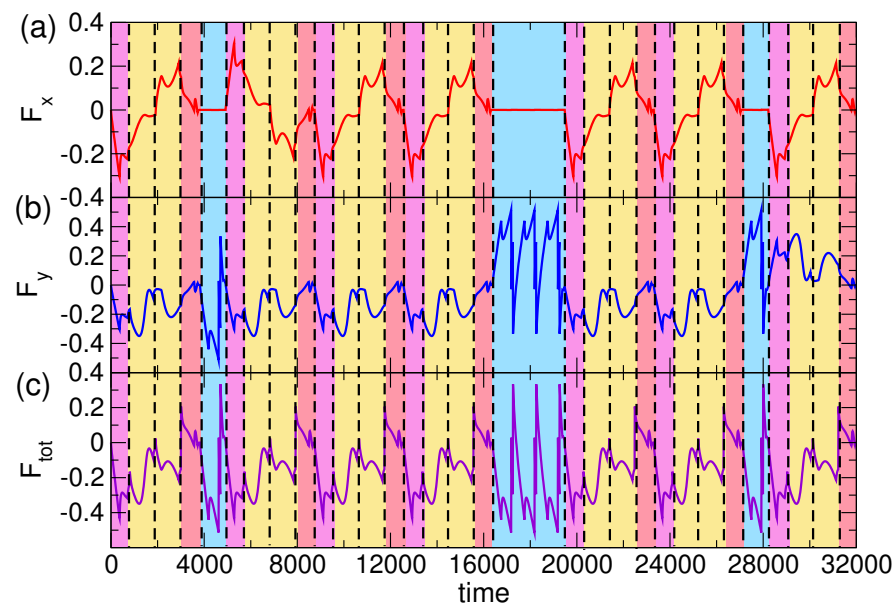

FIG. 25. Probe tip force signatures $F_{x}$ (a), $F_{y}$ (b), and $F_{\text {tot }}$ (c) vs time in simulation steps for the CNOT gate operation illustrated in Figs. 16 and 17. Magenta: capture; yellow: $\langle 10\rangle$ moves; pink: reverse capture; blue: repositioning of probe.

quantities for the $\langle 10\rangle$ braid operation. Each of these operations shows the reverse capture signature at the end; additionally, in the fourth stage of the $\langle 10\rangle$ braid, the signature associated with the indirect depinning of a vortex from a pinning site that is not immediately underneath the probe tip can be seen.

Figure 24 shows the probe tip force signals for the Hadamard gate and Fig. 25 shows the same for the CNOT gate. In both cases, signatures of the independent components of the gate moves can be seen. In actual experiments, the exact form of the pinning potential and probe tip interaction will likely differ from what we have assumed; however, there should still be distinct force signatures for the different categories of motion. In principle it would be possible to construct a library of the different force signatures that could be compared with the experimentally measured signals in order to confirm whether the operation was carried out successfully.

\section{DISCUSSION}

An important advantage of using a tip to move the vortices is that the same tip can also be used to measure the response of the Majorana fermion, as suggested

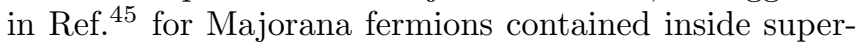
conducting vortices. A similar technique could be used to manipulate skyrmions containing Majorana fermion bound states 76 ; ; however, in this case, it is necessary to consider the dynamics carefully due to the strong Magnus component of the skyrmion motion, which is likely to affect how the skyrmions move under the influence of the tip and interact with pinning sites ${ }^{79}$. In this work, we considered moving the vortices with an MFM tip; however, there are also proposals for creating vortex logic devices using applied currents and specially structured pinning geometries 8485 . Alternative geometries such as these could also be used to achieve braiding operations.

\section{SUMMARY}

We have shown how to braid Majorana fermions in vortices on a periodic pinning array using an MFM tip. We specifically examine a superconductor coupled to a topological insulator with a square array of pinning sites in the form of blind holes at a magnetic field for which there is one vortex per pinning site. After demonstrating the fundamental operations of vortex capture, vortex motion, and probe tip relocation without motion of a vortex, we show how to perform vortex exchange and basic braiding operations by following a series of specific steps of probe tip motion. Based on the braiding operations, we construct Hadamard and CNOT gates, and show using numerical simulations that these gates can be operated successfully. In the presence of thermal noise, braids and motions involving the $\langle 11\rangle$ direction are not robust, so we utilize only the $\langle 10\rangle$ direction for maneuvering the vortices, while reserving $\langle 11\rangle$ motions for capture and reverse capture of vortices from the pinning sites. We show that the basic moves produce specific sequences of $x$ and $y$ force components on the probe tip, and that these sequences can be used to determine whether a gate operation has been completed successfully in experiment. Our results could be generalized for different pinning geometries, which could further optimize the robustness of the operations.

\section{ACKNOWLEDGMENTS}

This work was supported by the US Department of Energy through the Los Alamos National Laboratory. Los 
Alamos National Laboratory is operated by Triad National Security, LLC, for the National Nuclear Security
Administration of the U. S. Department of Energy (Contract No. 892333218NCA000001).
1 B. W. Gardner, J. C. Wynn, D. A. Bonn, R. X. Liang, W. N. Hardy, J. R. Kirtley, V. G. Kogan, and K. A. Moler, "Manipulation of single vortices in $\mathrm{YBa}_{2} \mathrm{Cu}_{3} \mathrm{O}_{6.354}$ with a locally applied magnetic field," Appl. Phys. Lett. 80, 1010-1012 (2002)

${ }^{2}$ E. W. J. Straver, J. E. Hoffman, O. M. Auslaender, D. Rugar, and Kathryn A. Moler, "Controlled manipulation of individual vortices in a superconductor," Appl. Phys. Lett. 93, $172514(2008)$

${ }^{3}$ O. M. Auslaender, L. Luan, E. W. J. Straver, J. E. Hoffman, N. C. Koshnick, E. Zeldov, D. A. Bonn, R. Liang, W. N. Hardy, and K. A. Moler, "Mechanics of individual isolated vortices in a cuprate superconductor," Nature Phys. 5, 35-39 (2009)

${ }^{4}$ L. Luan, O. M. Auslaender, D. A. Bonn, R. Liang, W. N. Hardy, and K. A. Moler, "Magnetic force microscopy study of interlayer kinks in individual vortices in the underdoped cuprate superconductor $\mathrm{YBa}_{2} \mathrm{Cu}_{3} \mathrm{O}_{6+x}$," Phys. Rev. B 79, 214530 (2009).

${ }^{5}$ N. Shapira, Y. Lamhot, O. Shpielberg, Y. Kafri, B. J. Ramshaw, D. A. Bonn, R. Liang, W. N. Hardy, and O. M. Auslaender, "Disorder-induced power-law response of a superconducting vortex on a plane," Phys. Rev. B 92, 100501 (2015)

${ }^{\circ}$ A. Kremen, S. Wissberg, N. Haham, E. Persky, Y. Frenkel, and B. Kalisky, "Mechanical control of individual superconducting vortices," Nano Lett. 16, 1626-1630 (2016)

7 J.-Y. Ge, V. N. Gladilin, J. Tempere, C. Xue, J. T. Devreese, J. Van de Vondel, Y. Zhou, and V. V. Moshchalkov, "Nanoscale assembly of superconducting vortices with scanning tunnelling microscope tip," Nature Commun. 7, 13880 (2016)

$\checkmark$ I. S. Veshchunov, W. Magrini, S. V. Mironov, A. G. Godin, J. B. Trebbia, A. I. Buzdin, Ph. Tamarat, and B. Lounis, "Optical manipulation of single flux quanta," Nature Commun. 7, 12801 (2016).

${ }^{y}$ C. J. Olson Reichhardt and M. B. Hastings, "Do vortices entangle?" Phys. Rev. Lett. 92, 157002 (2004).

10 Y. Kafri, D. R. Nelson, and A. Polkovnikov, "Unzipping flux lines from extended defects in type-II superconductors," Europhys. Lett. 73, 253-259 (2006).

11 Y. Kafri, D. R. Nelson, and A. Polkovnikov, "Unzipping vortices in type-II superconductors," Phys. Rev. B 76, 144501 (2007)

i2 C. Reichhardt, "Vortices wiggled and dragged," Nature Phys. 5, 15-16 (2009)

${ }^{13}$ C. J. Olson Reichhardt and C. Reichhardt, "Viscous decoupling transitions for individually dragged particles in systems with quenched disorder," Phys. Rev. E 78, 011402 (2008)

${ }^{14}$ C. J. Olson Reichhardt and C. Reichhardt, "Driving an individual vortex in the presence of a periodic pinning array," Physica C 470, 779-781 (2010).

15 X. Ma, C. J. O. Reichhardt, and C. Reichhardt, "Manipulation of individual superconducting vortices and stickslip motion in periodic pinning arrays," Phys. Rev. B 97, 214521 (2018)
16 J.-Y. Ge, V. N. Gladilin, J. Tempere, J. Devreese, and V. V. Moshchalkov, "Controlled generation of quantized vortex-antivortex pairs in a superconducting condensate," Nano Lett. 17, 5003-5007 (2017)

17 V. V. Dremov, S. Yu. Grebenchuk, A. G. Shishkin, D. S. Baranov, R. A. Hovhannisyan, O. V. Skryabina, I. A. Golovchanskiy, V. I. Chichkov, C. Brun, T. Cren, V. M. Krasnov, A. A. Golubov, D. Roditchev, and V. S. Stolyarov, "Local Josephson vortex generation and manipulation with a magnetic force microscope," Nature Commun. 10, 4009 (2019)

18 E. Majorana, "Symmetrical theory of electrons and positrons," Nuovo Cimento 14, 171-184 (1937).

19 L. P. Rokhinson, X. Liu, and J. K. Furdyna, "The fractional a.c. josephson effect in a semiconductorsuperconductor nanowire as a signature of Majorana particles," Nature Phys. 8, 795-799 (2012)

${ }^{20}$ V. Mourik, K. Zuo, S. M. Frolov, S. R. Plissard, E. P. A. M. Bakkers, and L. P. Kouwenhoven, "Signatures of Majorana fermions in hybrid superconductor-semiconductor nanowire devices," Science 336, 1003-1007 (2012)

21 C. W. J. Beenakker, "Search for Majorana fermions in superconductors," in Annual Review of Condensed Matter Physics, Vol. 4, Annual Review of Condensed Matter Physics, Vol. 4, edited by J. S. Langer (2013) pp. 113-136.

22 M. T. Deng, C. L. Yu, G. Y. Huang, M. Larsson, P. Caroff, and H. Q. Xu, "Parity independence of the zero-bias conductance peak in a nanowire based topological superconductor-quantum dot hybrid device," Sci. Rep. 4, 7261 (2014)

${ }^{23}$ S. Nadj-Perge, I. K. Drozdov, J. Li, H. Chen, S. Jeon, J. Seo, A. H. MacDonald, B. A. Bernevig, and A. Yazdani, "Observation of Majorana fermions in ferromagnetic atomic chains on a superconductor," Science 346, 602-607 (2014)

24 A. Banerjee, C. A. Bridges, J. Q. Yan, A. A. Aczel, L. Li, M. B. Stone, G. E. Granroth, M. D. Lumsden, Y. Yiu, J. Knolle, S. Bhattacharjee, D. L. Kovrizhin, R. Moessner, D. A. Tennant, D. G. Mandrus, and S. E. Nagler, "Proximate Kitaev quantum spin liquid behaviour in a honeycomb magnet," Nature Mater. 15, 733 (2016)

25 Q. L. He, L. Pan, A. L. Stern, E. C. Burks, X. Che, G. Yin, J. Wang, B. Lian, Q. Zhou, E. S. Choi, K. Murata, X. Kou, Z. Chen, T. Nie, Q. Shao, Y. Fan, S.-C. Zhang, K. Liu, J. Xia, and K. L. Wang, "Chiral Majorana fermion modes in a quantum anomalous Hall insulatorsuperconductor structure," Science 357, 294-299 (2017)

26 D. Wang, L. Kong, P. Fan, H. Chen, S. Zhu, W. Liu, L. Cao, Y. Sun, S. Du, J. Schneeloch, R. Zhong, G. Gu, L. Fu, H. Ding, and H.-J. Gao, "Evidence for Majorana bound states in an iron-based superconductor," Science 362, 333-335 (2018)

27 A. Das, Y. Ronen, Y. Most, Y. Oreg, M. Heiblum, and H. Shtrikman, "Zero-bias peaks and splitting in an Al-InAs nanowire topological superconductor as a signature of Majorana fermions," Nature Phys. 8, 887-895 (2012) 
28 S. Das Sarma, M. Freedman, and C. Nayak, "Majorana zero modes and topological quantum computation," NPJ Quant. Inform. 1, 15001 (2015)

${ }^{29}$ C. Nayak, S. H. Simon, A. Stern, M. Freedman, and S. Das Sarma, "Non-Abelian anyons and topological quantum computation," Rev. Mod. Phys. 80, 1083-1159 (2008).

30 A. Stern, "Non-Abelian states of matter," Nature (London) 464, 187-193 (2010)

${ }^{31}$ M. Leijnse and K. Flensberg, "Introduction to topological superconductivity and Majorana fermions," Semicond. Sci. Technol. 27, 124003 (2012)

${ }_{32}$ R. M. Lutchyn, E. P. A. M. Bakkers, L. P. Kouwenhoven, P. Krogstrup, C. M. Marcus, and Y. Oreg, "Majorana zero modes in superconductor-semiconductor heterostructures," Nature Rev. Mater. 3, 52-68 (2018).

33 M. Freedman, C. Nayak, and K. Walker, "Towards universal topological quantum computation in the $\nu=\frac{5}{2}$ fractional quantum Hall state," Phys. Rev. B 73, 245307 (2006)

${ }^{34}$ G. Moore and N. Read, "Nonabelions in the fractional quantum Hall-effect," Nucl. Phys. B 360, 362-396 (1991).

35 N. Read and D. Green, "Paired states of fermions in two dimensions with breaking of parity and time-reversal symmetries and the fractional quantum Hall effect," Phys. Rev. B 61, 10267-10297 (2000)

36 A. Yu. Kitaev, "Unpaired Majorana fermions in quantum wires," Phys.-Usp. 44, 131-136 (2001).

37 G. E. Volovik, The Universe in a Helium Droplet (Oxford University Press, Oxford, 2003).

38 J. Alicea, "New directions in the pursuit of Majorana fermions in solid state systems," Rep. Prog. Phys. 75, 076501 (2012)

${ }^{39}$ M. Stone and S.-B. Chung, "Fusion rules and vortices in $p_{x}+i p_{y}$ superconductors," Phys. Rev. B 73, 014505 (2006)

40 D. A. Ivanov, "Non-Abelian statistics of half-quantum vortices in $p$-wave superconductors," Phys. Rev. Lett. 86, 268-271 (2001).

${ }^{41}$ L. Fu and C. L. Kane, "Superconducting proximity effect and Majorana fermions at the surface of a topological insulator," Phys. Rev. Lett. 100, 096407 (2008).

42 H.-H. Sun, K.-W. Zhang, L.-H. Hu, C. Li, G.-Y. Wang, H.-Y. Ma, Z.-A. Xu, C.-L. Gao, D.-D. Guan, Y.-Y. Li, C. Liu, D. Qian, Y. Zhou, L. Fu, S.-C. Li, F.-C. Zhang, and J.-F. Jia, "Majorana zero mode detected with spin selective Andreev reflection in the vortex of a topological superconductor," Phys. Rev. Lett. 116, 257003 (2016)

43 H.-H. Sun and J.-F. Jia, "Detection of Majorana zero mode in the vortex," NPJ Quantum Mater. 2, 34 (2017).

${ }^{4}$ H.-D. Wu and T. Zhou, "Vortex pinning by the point potential in topological superconductors: A scheme for braiding Majorana bound states," Phys. Rev. B 96, 184508 (2017)

40 B. H. November, J. D. Sau, J. R. Williams, and J. E. Hoffman, "Scheme for Majorana manipulation using magnetic force microscopy," (2019), arXiv:1905.09792.

${ }^{46}$ H. Polshyn, T. Naibert, and R. Budakian, "Manipulating multivortex states in superconducting structures," Nano Lett. 19, 5476-5482 (2019)

4. T. Posske, C.-K. Chiu, and M. Thorwart, "Robustly emulating vortex Majorana braiding in a finite time," (2019), arXiv:1908.03576

48 Q.-F. Liang, Z. Wang, and X. Hu, "Manipulation of Majorana fermions by point-like gate voltage in the vortex state of a topological superconductor," EPL 99, 50004 (2012)
${ }^{49}$ C. W. J. Beenakker, "Search for non-Abelian Majorana braiding statistics in superconductors," (2019), arXiv:1907.06497.

50 A. Stern and E. Berg, "Fractional Josephson vortices and braiding of Majorana zero modes in planar superconductor-semiconductor heterostructures," Phys. Rev. Lett. 122, 107701 (2019).

${ }^{51}$ C. W. J. Beenakker, P. Baireuther, Y. Herasymenko, I. Adagideli, L. Wang, and A. R. Akhmerov, "Deterministic creation and braiding of chiral edge vortices," Phys. Rev. Lett. 122, 146803 (2019).

${ }^{2}$ H. S. Røising, R. Ilan, T. Meng, S. H. Simon, and F. Flicker, "Finite temperature effects on Majorana bound states in chiral $p$-wave superconductors," SciPost Phys. 6, 055 (2019).

5 M. Baert, V. V. Metlushko, R. Jonckheere, V. V. Moshchalkov, and Y. Bruynseraede, "Composite flux-line lattices stabilized in superconducting films by a regular array of artificial defects," Phys. Rev. Lett. 74, 3269-3272 (1995)

${ }^{54}$ K. Harada, O. Kamimura, H. Kasai, T. Matsuda, A. Tonomura, and V. V. Moshchalkov, "Direct observation of vortex dynamics in superconducting films with regular arrays of defects," Science 274, 1167-1170 (1996)

55 J. I. Martín, M. Vélez, J. Nogués, and I. K. Schuller, "Flux pinning in a superconductor by an array of submicrometer magnetic dots," Phys. Rev. Lett. 79, 1929-1932 (1997)

56 G. R. Berdiyorov, M. V. Milošević, and F. M. Peeters, "Novel commensurability effects in superconducting films with antidot arrays," Phys. Rev. Lett. 96, 207001 (2006)

57 M. Kemmler, C. Gürlich, A. Sterck, H. Pöhler, M. Neuhaus, M. Siegel, R. Kleiner, and D. Koelle, "Commensurability effects in superconducting $\mathrm{Nb}$ films with quasiperiodic pinning arrays," Phys. Rev. Lett. 97, 147003 (2006)

58 C. C. de Souza Silva, A. V. Silhanek, J. Van de Vondel, W. Gillijns, V. Metlushko, B. Ilic, and V. V. Moshchalkov, "Dipole-induced vortex ratchets in superconducting films with arrays of micromagnets," Phys. Rev. Lett. 98, 117005 (2007)

59 S. Goldberg, Y. Segev, Y. Myasoedov, I. Gutman, N. Avraham, M. Rappaport, E. Zeldov, T. Tamegai, C. W. Hicks, and K. A. Moler, "Mott insulator phases and first-order melting in $\mathrm{Bi}_{2} \mathrm{Sr}_{2} \mathrm{CaCu}_{2} \mathrm{O}_{8+\delta}$ crystals with periodic surface holes," Phys. Rev. B 79, 064523 (2009).

60 M. Kemmler, D. Bothner, K. Ilin, M. Siegel, R. Kleiner, and D. Koelle, "Suppression of dissipation in Nb thin films with triangular antidot arrays by random removal of pinning sites," Phys. Rev. B 79, 184509 (2009)

61 J. Gutierrez, A. V. Silhanek, J. Van de Vondel, W. Gillijns, and V. V. Moshchalkov, "Transition from turbulent to nearly laminar vortex flow in superconductors with periodic pinning," Phys. Rev. B 80, 140514 (2009).

62 A. Libál, C. J. Olson Reichhardt, and C. Reichhardt, "Creating artificial ice states using vortices in nanostructured superconductors," Phys. Rev. Lett. 102, 237004 (2009)

63 A. Hoffmann, P. Prieto, V. Metlushko, and I. K. Schuller, "Superconducting vortex pinning with magnetic dots: Does size and magnetic configuration matter?" J. Supercond. Novel Mag. 25, 2187-2191 (2012).

${ }^{64}$ I. Swiecicki, C. Ulysse, T. Wolf, R. Bernard, N. Bergeal, J. Briatico, G. Faini, J. Lesueur, and Javier E. Villegas, "Strong field-matching effects in superconducting $\mathrm{YBa}_{2} \mathrm{Cu}_{3} \mathrm{O}_{7-\delta}$ films with vortex energy landscapes en- 
gineered via masked ion irradiation," Phys. Rev. B 85, 224502 (2012)

os M. L. Latimer, G. R. Berdiyorov, Z. L. Xiao, F. M. Peeters, and W. K. Kwok, "Realization of artificial ice systems for magnetic vortices in a superconducting MoGe thin film with patterned nanostructures," Phys. Rev. Lett. 111, 067001 (2013)

कै Y. L. Wang, M. L. Latimer, Z. L. Xiao, R. Divan, L. E. Ocola, G. W. Crabtree, and W. K. Kwok, "Enhancing the critical current of a superconducting film in a wide range of magnetic fields with a conformal array of nanoscale holes," Phys. Rev. B 87, 220501 (2013)

67 D. Ray, C. Reichhardt, and C. J. Olson Reichhardt, "Pinning, ordering, and dynamics of vortices in conformal crystal and gradient pinning arrays," Phys. Rev. B 90, 094502 (2014)

68 J. Trastoy, M. Malnou, C. Ulysse, R. Bernard, N. Bergeal, G. Faini, J. Lesueur, J. Briatico, and J. E. Villegas, "Freezing and thawing of artificial ice by thermal switching of geometric frustration in magnetic flux lattices," Nature Nanotechnol. 9, 710-715 (2014).

${ }^{\circ y}$ I. A. Sadovskyy, Y. L. Wang, Z.-L. Xiao, W.-K. Kwok, and A. Glatz, "Effect of hexagonal patterned arrays and defect geometry on the critical current of superconducting films," Phys. Rev. B 95, 075303 (2017).

70 G. Zechner, F. Jausner, L. T. Haag, W. Lang, M. Dosmailov, M. A. Bodea, and J. D. Pedarnig, "Hysteretic vortex-matching effects in high- $T_{c}$ superconductors with nanoscale periodic pinning landscapes fabricated by He ion-beam projection," Phys. Rev. Applied 8, 014021 (2017)

${ }^{7}$ C. Xue, J.-Y. Ge, A. He, V. S. Zharinov, V. V. Moshchalkov, Y. H. Zhou, A. V. Silhanek, and J. Van de Vondel, "Tunable artificial vortex ice in nanostructured superconductors with a frustrated kagome lattice of paired antidots," Phys. Rev. B 97, 134506 (2018).

72 J.-Y. Ge, V. N. Gladilin, J. Tempere, J. T. Devreese, and V. V. Moshchalkov, "Tunable and switchable magnetic dipole patterns in nanostructured superconductors," Nature Commun. 9, 2576 (2018)

13 Y.-L. Wang, X. Ma, J. Xu, Z.-L. Xiao, A. Snezhko, R. Divan, L. E. Ocola, J. E. Pearson, B. Jánko, and W.-K. Kwok, "Switchable geometric frustration in an artificial- spin-ice-superconductor heterosystem," Nature Nanotechnol. 13, 560 (2018).

${ }^{4}$ L. S. Georgiev, "Topologically protected gates for quantum computation with non-Abelian anyons in the Pfaffian quantum Hall state," Phys. Rev. B 74, 235112 (2006).

75 L. S. Georgiev, "Towards a universal set of topologically protected gates for quantum computation with Pfaffian qubits," Nucl. Phys. B 789, 552-590 (2008)

76 G. Yang, P. Stano, J. Klinovaja, and D. Loss, "Majorana bound states in magnetic skyrmions," Phys. Rev. B 93, 224505 (2016)

7 U. Güngördü, S. Sandhoefner, and A. A. Kovalev, "Stabilization and control of Majorana bound states with elongated skyrmions," Phys. Rev. B 97, 115136 (2018)

78 S. Rex, I. V. Gornyi, and A. D. Mirlin, "Majorana bound states in magnetic skyrmions imposed onto a superconductor," (2019), arXiv:1904.04177

79 C. Reichhardt, D. Ray, and C. J. O. Reichhardt, "Nonequilibrium phases and segregation for skyrmions on periodic pinning arrays," Phys. Rev. B 98, 134418 (2018)

80 C. Hanneken, A. Kubetzka, K. von Bergmann, and R. Wiesendanger, "Pinning and movement of individual nanoscale magnetic skyrmions via defects," New J. Phys. 18, 055009 (2016).

${ }^{81}$ R. M. Menezes, J. F. S. Neto, C. C. de Souza Silva, and M. V. Milošević, "Manipulation of magnetic skyrmions by superconducting vortices in ferromagnet-superconductor heterostructures," Phys. Rev. B 100, 014431 (2019).

82 M. Cheng, R. M. Lutchyn, V. Galitski, and S. Das Sarma, "Splitting of Majorana-Fermion modes due to intervortex tunneling in a $p_{x}+i p_{y}$ superconductor," Phys. Rev. Lett. 103, 107001 (2009).

${ }_{83}$ M. Cheng, R. M. Lutchyn, V. Galitski, and S. Das Sarma, "Tunneling of anyonic Majorana excitations in topological superconductors," Phys. Rev. B 82, 094504 (2010).

84 M. B. Hastings, C. J. Olson Reichhardt, and C. Reichhardt, "Ratchet cellular automata," Phys. Rev. Lett. 90, 247004 (2003)

${ }^{85}$ M. V. Milosevic, G. R. Berdiyorov, and F. M. Peeters, "Fluxonic cellular automata," Appl. Phys. Lett. 91, 212501 (2007) 